\title{
TIME-INTEGRATED SEARCHES FOR POINT-LIKE SOURCES OF NEUTRINOS WITH THE 40-STRING IceCube DETECTOR
}

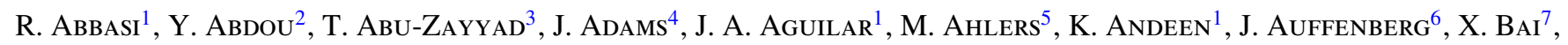
M. BaKer ${ }^{1}$, S. W. Barwick ${ }^{8}$, R. BaY ${ }^{9}$, J. L. Bazo Alba ${ }^{10}$, K. Beattie ${ }^{11}$, J. J. Beatty ${ }^{12,13}$, S. Bechet ${ }^{14}$, J. K. Becker ${ }^{15}$, K.-H. Becker ${ }^{6}$, M. L. Benabderrahmane ${ }^{10}$, S. BenZvi ${ }^{1}$, J. Berdermann ${ }^{10}$, P. Berghaus ${ }^{1}$, D. Berley ${ }^{16}$, E. Bernardini ${ }^{10}$, D. Bertrand ${ }^{14}$, D. Z. Besson ${ }^{17}$, M. BissoK ${ }^{18}$, E. Blaufuss ${ }^{16}$, J. Blumenthal ${ }^{18}$, D. J. Boersma ${ }^{18}$, C. Bohm ${ }^{19}$, D. Bose ${ }^{20}$,

S. Böser ${ }^{21}$, O. Botner ${ }^{22}$, J. Braun ${ }^{1}$, A. M. Brown ${ }^{4}$, S. Buitink ${ }^{11}$, M. Carson ${ }^{2}$, D. Chirkin ${ }^{1}$, B. Christy ${ }^{16}$, J. Clem $^{7}$,

F. Clevermann ${ }^{23}$, S. Cohen ${ }^{24}$, C. Colnard ${ }^{25}$, D. F. Cowen ${ }^{26,27}$, M. V. D'Agostino ${ }^{9}$, M. Danninger ${ }^{19}$, J. Daughhetee ${ }^{28}$,

J. C. Davis ${ }^{12}$, C. De Clercq ${ }^{20}$, L. Demirörs ${ }^{24}$, O. Depaepe ${ }^{20}$, F. Descamps ${ }^{2}$, P. Desiati ${ }^{1}$, G. De Vries-Uiterweerd ${ }^{2}$,

T. DeYoung ${ }^{26}$, J. C. Díaz-Vélez ${ }^{1}$, M. Dierckxsens ${ }^{14}$, J. Dreyer ${ }^{15}$, J. P. Dumm ${ }^{1}$, R. Ehrlich ${ }^{16}$, J. Eisch ${ }^{1}$, R. W. Ellsworth ${ }^{16}$, O. EngdegåRD ${ }^{22}$, S. Euler ${ }^{18}$, P. A. Evenson ${ }^{7}$, O. Fadiran ${ }^{29}$, A. R. FAzely ${ }^{30}$, A. Fedynitch ${ }^{15}$, T. Feusels ${ }^{2}$, K. Filimonov ${ }^{9}$, C. Finley ${ }^{19}$, M. M. Foerster ${ }^{26}$, B. D. Fox ${ }^{26}$, A. FrancKowiak ${ }^{21}$, R. Franke $^{10}$, T. K. Gaisser ${ }^{7}$, J. Gallagher ${ }^{31}$, M. Geisler ${ }^{18}$,

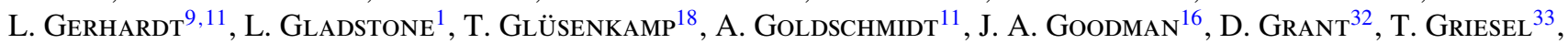

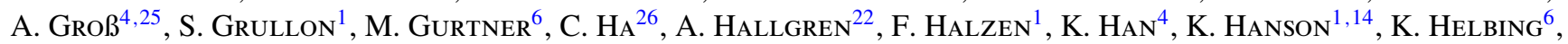
P. Herquet ${ }^{34}$, S. Hickford ${ }^{4}$, G. C. Hill ${ }^{1}$, K. D. Hoffman ${ }^{16}$, A. Homeier ${ }^{21}$, K. Hoshina ${ }^{1}$, D. Hubert ${ }^{20}$, W. Huelsnitz ${ }^{16}$, J.-P. Hül $\beta^{18}$, P. O. Hulth ${ }^{19}$, K. Hultqvist ${ }^{19}$, S. Hussain ${ }^{7}$, A. Ishihara ${ }^{35}$, J. JACOBSEN ${ }^{1}$, G. S. Japaridze ${ }^{29}$, H. Johansson ${ }^{19}$,

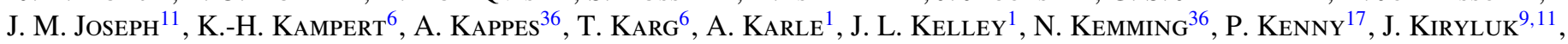
F. $\operatorname{Kislat~}^{10}$, S. R. KLein ${ }^{9,11}$, J.-H. KöHNe ${ }^{23}$, G. Kohnen ${ }^{34}$, H. Kolanoski ${ }^{36}$, L. KöPKE ${ }^{33}$, D. J. Koskinen ${ }^{26}$, M. KowalsKi ${ }^{21}$,

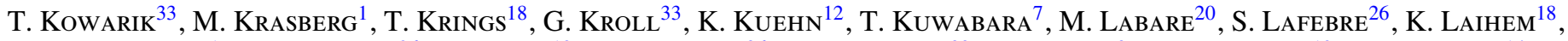
H. Landsman ${ }^{1}$, M. J. Larson ${ }^{26}$, R. Lauer ${ }^{10}$, R. LehmanN ${ }^{36}$, J. LÜNemanN ${ }^{33}$, J. MadSen ${ }^{3}$, P. MajumdaR ${ }^{10}$, A. Marotta ${ }^{14}$,

R. Maruyama ${ }^{1}$, K. Mase ${ }^{35}$, H. S. Matis ${ }^{11}$, M. MatusiK ${ }^{6}$, K. Meagher ${ }^{16}$, M. Merck ${ }^{1}$, P. Mészáros ${ }^{26,27}$, T. Meures ${ }^{18}$,

E. Middell ${ }^{10}$, N. Milke ${ }^{23}$, J. Miller ${ }^{22}$, T. Montaruli ${ }^{1},{\text { R. } \text { Morse }^{1} \text {, S. M. Movit }}^{27}$, R. Nahnhauer ${ }^{10}$, J. W. Nam ${ }^{8}$,

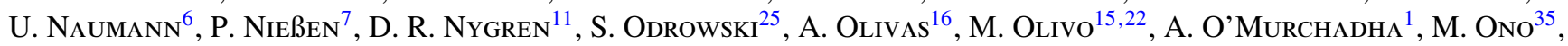
S. Panknin ${ }^{21}$, L. Paul ${ }^{18}$, C. Pérez de los $\operatorname{Heros}^{22}$, J. Petrovic ${ }^{14}$, A. Piegsa ${ }^{33}$, D. Pieloth ${ }^{23}$, R. Porrata ${ }^{9}$, J. Posselt ${ }^{6}$, P. B. Price 9 , M. Prikockis ${ }^{26}$, G. T. Przybylski ${ }^{11}$, K. Rawlins ${ }^{37}$, P. RedL ${ }^{16}$, E. ResConi ${ }^{25}$, W. Rhode ${ }^{23}$, M. Ribordy ${ }^{24}$,

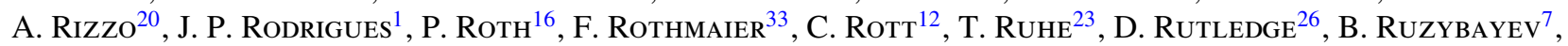
D. Ryckbosch ${ }^{2}$, H.-G. SANDeR ${ }^{33}$, M. SANTANDER ${ }^{1}$, S. SARKaR ${ }^{5}$, K. Schatto ${ }^{33}$, S. Schlenstedt ${ }^{10}$, T. Schmidt ${ }^{16}$, A. Schukraft ${ }^{18}$, A. Schultes ${ }^{6}$, O. Schulz ${ }^{25}$, M. Schunck ${ }^{18}$, D. Seckel ${ }^{7}$, B. Semburg ${ }^{6}$, S. H. Seo ${ }^{19}$, Y. Sestayo ${ }^{25}$, S. Seunarine ${ }^{38}$, A. Silvestri ${ }^{8}$, K. Singh ${ }^{20}$, A. SlipaK ${ }^{26}$, G. M. SpiczaK ${ }^{3}$, C. Spiering ${ }^{10}$, M. Stamatikos ${ }^{12,39}$, T. Stanev ${ }^{7}$, G. Stephens ${ }^{26}$, T. Stezelberger ${ }^{11}$, R. G. Stokstad ${ }^{11}$, S. Stoyanov ${ }^{7}$, E. A. Strahler ${ }^{20}$, T. Straszheim ${ }^{16}$, G. W. Sullivan ${ }^{16}$, Q. Swillens ${ }^{14}$, H. TaAvola ${ }^{22}$, I. Taboada ${ }^{28}$, A. Tamburro ${ }^{3}$, O. Tarasova ${ }^{10}$, A. Tepe ${ }^{28}$, S. Ter-Antonyan $^{30}$, S. TilavV $^{7}$,

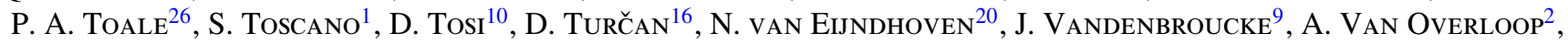
J. van Santen ${ }^{1}$, M. Vehring ${ }^{18}$, M. Voge ${ }^{25}$, B. Voigt ${ }^{10}$, C. WalcK $^{19}$, T. Waldenmaier $^{36}$, M. WallrafF $^{18}$, M. Walter $^{10}$, Ch. Weaver ${ }^{1}$, C. Wendt ${ }^{1}$, S. Westerhoff ${ }^{1}$, N. Whitehorn ${ }^{1}$, K. Wiebe ${ }^{33}$, C. H. Wiebusch ${ }^{18}$, D. R. Williams ${ }^{40}$,

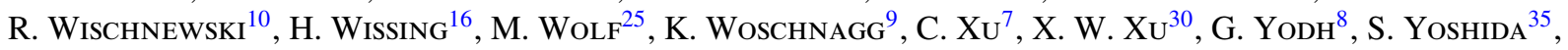
AND P. ZARZHITSKY ${ }^{40}$

(ICECube Collaboration)

\author{
${ }^{1}$ Department of Physics, University of Wisconsin, Madison, WI 53706, USA \\ 2 Department of Subatomic and Radiation Physics, University of Gent, B-9000 Gent, Belgium \\ ${ }^{3}$ Department of Physics, University of Wisconsin, River Falls, WI 54022, USA \\ ${ }^{4}$ Department of Physics and Astronomy, University of Canterbury, Private Bag 4800, Christchurch, New Zealand \\ ${ }^{5}$ Department of Physics, University of Oxford, 1 Keble Road, Oxford OX1 3NP, UK \\ ${ }^{6}$ Department of Physics, University of Wuppertal, D-42119 Wuppertal, Germany \\ ${ }^{7}$ Bartol Research Institute and Department of Physics and Astronomy, University of Delaware, Newark, DE 19716, USA \\ ${ }^{8}$ Department of Physics and Astronomy, University of California, Irvine, CA 92697, USA \\ ${ }^{9}$ Department of Physics, University of California, Berkeley, CA 94720, USA \\ ${ }^{10}$ DESY, D-15735 Zeuthen, Germany \\ ${ }^{11}$ Lawrence Berkeley National Laboratory, Berkeley, CA 94720, USA \\ ${ }^{12}$ Department of Physics and Center for Cosmology and Astro-Particle Physics, Ohio State University, Columbus, OH 43210, USA \\ ${ }_{14}^{13}$ Department of Astronomy, Ohio State University, Columbus, OH 43210, USA \\ ${ }^{14}$ Université Libre de Bruxelles, Science Faculty CP230, B-1050 Brussels, Belgium \\ ${ }^{15}$ Fakultät für Physik \& Astronomie, Ruhr-Universität Bochum, D-44780 Bochum, Germany \\ ${ }^{16}$ Department of Physics, University of Maryland, College Park, MD 20742, USA \\ ${ }^{17}$ Department of Physics and Astronomy, University of Kansas, Lawrence, KS 66045, USA \\ 18 III. Physikalisches Institut, RWTH Aachen University, D-52056 Aachen, Germany \\ ${ }^{19}$ Oskar Klein Centre and Deptartment of Physics, Stockholm University, SE-10691 Stockholm, Sweden \\ ${ }^{20}$ Vrije Universiteit Brussel, Dienst ELEM, B-1050 Brussels, Belgium \\ ${ }^{21}$ Physikalisches Institut, Universität Bonn, Nussallee 12, D-53115 Bonn, Germany \\ ${ }^{22}$ Department of Physics and Astronomy, Uppsala University, Box 516, S-75120 Uppsala, Sweden \\ ${ }^{23}$ Department of Physics, TU Dortmund University, D-44221 Dortmund, Germany \\ ${ }^{24}$ Laboratory for High Energy Physics, École Polytechnique Fédérale, CH-1015 Lausanne, Switzerland
}




\author{
${ }^{25}$ Max-Planck-Institut für Kernphysik, D-69177 Heidelberg, Germany \\ ${ }^{26}$ Department of Physics, Pennsylvania State University, University Park, PA 16802, USA \\ ${ }^{27}$ Department of Astronomy and Astrophysics, Pennsylvania State University, University Park, PA 16802, USA \\ ${ }^{28}$ School of Physics and Center for Relativistic Astrophysics, Georgia Institute of Technology, Atlanta, GA 30332, USA \\ ${ }^{29}$ CTSPS, Clark-Atlanta University, Atlanta, GA 30314, USA \\ ${ }^{30}$ Department of Physics, Southern University, Baton Rouge, LA 70813, USA \\ ${ }^{31}$ Department of Astronomy, University of Wisconsin, Madison, WI 53706, USA \\ ${ }^{32}$ Department of Physics, University of Alberta, Edmonton, Alberta, T6G 2G7, Canada \\ ${ }^{33}$ Institute of Physics, University of Mainz, Staudinger Weg 7, D-55099 Mainz, Germany \\ ${ }^{34}$ Université de Mons, 7000 Mons, Belgium \\ ${ }^{35}$ Department of Physics, Chiba University, Chiba 263-8522, Japan \\ ${ }^{36}$ Institut für Physik, Humboldt-Universität zu Berlin, D-12489 Berlin, Germany \\ ${ }^{37}$ Department of Physics and Astronomy, University of Alaska Anchorage, 3211 Providence Dr., Anchorage, AK 99508, USA \\ ${ }^{38}$ Department of Physics, University of the West Indies, Cave Hill Campus, Bridgetown BB11000, Barbados \\ ${ }^{39}$ NASA Goddard Space Flight Center, Greenbelt, MD 20771, USA \\ ${ }^{40}$ Department of Physics and Astronomy, University of Alabama, Tuscaloosa, AL 35487, USA \\ Received 2010 November 30; accepted 2011 February 27; published 2011 April 8
}

\begin{abstract}
We present the results of time-integrated searches for astrophysical neutrino sources in both the northern and southern skies. Data were collected using the partially completed IceCube detector in the 40-string configuration recorded between 2008 April 5 and 2009 May 20, totaling 375.5 days livetime. An unbinned maximum likelihood ratio method is used to search for astrophysical signals. The data sample contains 36,900 events: 14,121 from the northern sky, mostly muons induced by atmospheric neutrinos, and 22,779 from the southern sky, mostly high-energy atmospheric muons. The analysis includes searches for individual point sources and stacked searches for sources in a common class, sometimes including a spatial extent. While this analysis is sensitive to $\mathrm{TeV}-\mathrm{PeV}$ energy neutrinos in the northern sky, it is primarily sensitive to neutrinos with energy greater than about $1 \mathrm{PeV}$ in the southern sky. No evidence for a signal is found in any of the searches. Limits are set for neutrino fluxes from astrophysical sources over the entire sky and compared to predictions. The sensitivity is at least a factor of two better than previous searches (depending on declination), with $90 \%$ confidence level muon neutrino flux upper limits being between $E^{2} d \Phi / d E \sim 2-200 \times 10^{-12} \mathrm{TeV} \mathrm{cm}^{-2} \mathrm{~s}^{-1}$ in the northern sky and between 3-700 $\times 10^{-12} \mathrm{TeV} \mathrm{cm}^{-2} \mathrm{~s}^{-1}$ in the southern sky. The stacked source searches provide the best limits to specific source classes. The full IceCube detector is expected to improve the sensitivity to $d \Phi / d E \propto E^{-2}$ sources by another factor of two in the first year of operation.
\end{abstract}

Key words: astroparticle physics - cosmic rays - neutrinos

Online-only material: color figures

\section{INTRODUCTION}

Neutrino astronomy is tightly connected to cosmic ray (CR) and gamma-ray astronomy, since neutrinos likely share their origins with these other messengers. With a possible exception at the highest observed energies, CRs propagate diffusively losing directional information due to magnetic fields, and both CRs and gamma rays at high energies are absorbed due to interactions on photon backgrounds. Neutrinos, on the other hand, are practically unabsorbed en route and travel directly from cosmological sources to the Earth. Neutrinos are therefore fundamental to understanding CR acceleration processes up to the highest energies, and the detection of astrophysical neutrino sources could unveil the origins of hadronic CR acceleration. Whether or not gamma-ray energy spectra above about $10 \mathrm{TeV}$ can be accounted for by only inverse Compton processes is still an open question. Some observations suggest contributions from hadronic acceleration processes (Morlino et al. 2009; Boettcher et al. 2009). Acceleration of CRs is thought to take place in shocks in supernova remnants (SNRs) or in jets produced in the vicinity of accretion disks by processes which are not fully understood. Black holes in active galactic nuclei (AGNs), galactic micro-quasars and magnetars, or disruptive phenomena such as collapsing stars or binary mergers leading to gamma-ray bursts (GRBs), all characterized by relativistic outflows, could also be powerful accelerators. The canonical model for acceleration of CRs is the Fermi model (Fermi 1949), called first-order Fermi acceleration when applied to nonrelativistic shock fronts. This model naturally gives a CR energy spectrum similar to $d \Phi / d E \propto E^{-2}$ at the source. The neutrinos, originating in $\mathrm{CR}$ interactions near the source, are expected to follow a similar energy spectrum. More recently, models such as those in Caprioli et al. (2010) can yield significantly harder source spectra. In the framework of these models, it is possible to account for galactic $\mathrm{CR}$ acceleration to energies up to the knee, at about $Z \times 4 \times 10^{15} \mathrm{eV}$, where $Z$ is the atomic number of the CR. Extragalactic sources, on the other hand, are believed to be responsible for ultra-high-energy CRs observed up to about $10^{20} \mathrm{eV}$.

The concept of a neutrino telescope as a three-dimensional matrix of photomultiplier tubes (PMTs) was originally proposed by Markov \& Zheleznykh (1961). These sensors detect the Cerenkov light induced by relativistic charged particles passing through a transparent and dark medium such as deep water or the Antarctic ice sheet. The depth of these detectors helps to filter out the large number of atmospheric muons, making it possible to detect the rarer neutrino events. The direction and energy of particles are reconstructed using the arrival time and number of the Cerenkov photons. High-energy muon-neutrino interactions produce muons that can travel many kilometers. On average, the muons scatter $<0.1$ with respect to the original neutrino direction for $E_{v}>10 \mathrm{TeV}$. The first cubic-kilometer 
neutrino telescope, IceCube, is being completed at the South Pole. IceCube has a large target mass. This gives it excellent sensitivity to astrophysical neutrinos, enabling it to test many theoretical predictions.

Reviews on neutrino sources and telescopes can be found in Anchordoqui \& Montaruli (2010), Chiarusi \& Spurio (2010), Becker (2008), Lipari (2006), Bednarek et al. (2005), Halzen \& Hooper (2002), Learned \& Mannheim (2000), and Gaisser et al. (1995). Recent results on searches for neutrino sources have been published by IceCube in the 22-string configuration (Abbasi et al. 2009a, 2009b), AMANDA-II (Abbasi et al. 2009c), Super-Kamiokande (Thrane et al. 2009), and MACRO (Ambrosio et al. 2001).

This paper is structured as follows: Section 2 describes the detector. The data sample and cut parameters are discussed in Section 3, along with the simulation. In Section 4, the detector performance is characterized for searches. Section 5 describes the unbinned maximum likelihood search method, and in Section 6 the point-source and stacking searches are discussed. After discussing the systematic errors in Section 7, the results are presented in Section 8. Section 9 discusses the impact of our results on various possible neutrino emission models, and Section 10 offers some conclusions.

\section{DETECTOR AND DATA SAMPLE}

The IceCube Neutrino Observatory is composed of a deep array of 86 strings holding 5160 digital optical modules (DOMs) deployed between 1.45 and $2.45 \mathrm{~km}$ below the surface of the South Pole ice. The strings are typically separated by about $125 \mathrm{~m}$ with DOMs separated vertically by about $17 \mathrm{~m}$ along each string. IceCube construction started with the first string installed in the 2005-2006 austral summer (Achterberg et al. 2006a) and was completed in 2010 December. Six of the strings in the final detector use high quantum efficiency DOMs and a spacing of about $70 \mathrm{~m}$ horizontally and $7 \mathrm{~m}$ vertically. Two more strings have standard IceCube DOMs and $7 \mathrm{~m}$ vertical spacing but an even smaller horizontal spacing of $42 \mathrm{~m}$. These eight strings along with seven neighboring standard strings make up DeepCore, designed to enhance the physics performance of IceCube below $1 \mathrm{TeV}$. The observatory also includes a surface array, IceTop, for extensive air shower measurements on the composition and spectrum of CRs.

Each DOM consists of a $25 \mathrm{~cm}$ diameter Hamamatsu PMT (Abbasi et al. 2010a), electronics for waveform digitization (Abbasi et al. 2009d), and a spherical, pressure-resistant glass housing. A single Cerenkov photon arriving at a DOM can produce a photoelectron, which is called a hit if the analog output of the PMT exceeds a threshold equivalent to 0.25 of the average single photoelectron (SPE) charge. The waveform of the PMT total charge is digitized and sent to the surface if hits are in coincidence with at least one other hit in the nearest or next-tonearest neighboring DOMs within \pm 1000 ns. Hits that satisfy this condition are called local coincidence hits. The waveforms can contain multiple hits. The total number of photoelectrons and their arrival times are extracted with an iterative Bayesianbased unfolding algorithm. This algorithm uses the template shape representing an average hit.

Forty strings of IceCube were in operation from 2008 April 5 to 2009 May 20. The layout of these strings in relation to the final 86-string IceCube configuration is shown in Figure 1. Over the entire period the detector ran with an uptime of $92 \%$, yielding 375.5 days of total exposure. Dead time is mainly due to test runs during and after the construction season dedicated to

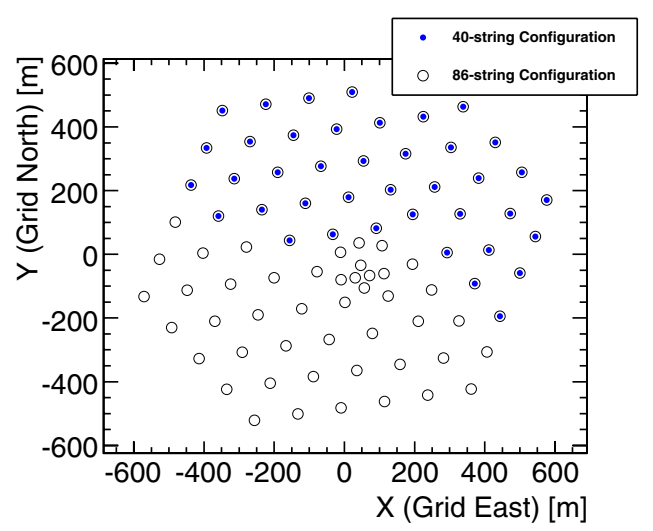

Figure 1. Overhead view of the 40-string configuration, along with additional strings that will make up the complete IceCube detector.

(A color version of this figure is available in the online journal.)

calibrating the additional strings and upgrading data acquisition systems.

IceCube uses a simple multiplicity trigger, requiring local coincidence hits in eight DOMs within $5 \mu$ s. Once the trigger condition is met, local coincidence hits within a readout window $\pm 10 \mu$ s are recorded, and overlapping readout windows are merged together. IceCube triggers primarily on down-going muons at a rate of about $950 \mathrm{~Hz}$ in this (40-string) configuration. Variations in the trigger rate by about $\pm 10 \%$ are due to seasonal changes affecting development of CR showers and muon production in the atmosphere, with higher rates during the austral summer (Tilav et al. 2010).

\section{DATA AND SIMULATION}

\subsection{Data Sample}

Traditional astrophysical neutrino point-source searches have used the Earth to block all upward traveling (up-going) particles except muons induced by neutrinos, as in Abbasi et al. (2009b). There remains a background of up-going muons from neutrinos, which are created in CR air showers and can penetrate the entire Earth. These atmospheric neutrinos have a softer energy spectrum than many expectations for astrophysical neutrinos. The measurement of the atmospheric neutrino spectrum for the 40-string detector is discussed in Abbasi et al. (2010b). A large number of muons produced in CR showers in the atmosphere and moving downward through the detector (downgoing) are initially misreconstructed as up-going. These mask the neutrino events until quality selections are made, leaving only a small residual of misreconstructed events.

The down-going region is dominated by atmospheric muons that also have a softer spectrum compared to muons induced by astrophysical neutrinos. At present, this large background reduces the IceCube sensitivity to neutrino sources in the southern sky in the sub-PeV energy region. While veto techniques are in development which will enable larger detector configurations to isolate neutrino-induced events starting within the detector, point-source searches can meanwhile be extended to the downgoing region if the softer-spectrum atmospheric muon background is reduced by an energy selection. This was done for the first time using the previous 22-string configuration of IceCube (Abbasi et al. 2009a), extending IceCube's field of view to $-50^{\circ}$ declination. In this paper, we extend the field of view to $-85^{\circ}$ declination (the exclusion between $-85^{\circ}$ and $-90^{\circ}$ is due to the use of scrambled data for background estimation in the analysis, 
Table 1

Number of Events at Each Processing Level for the 375.5 days of Livetime

\begin{tabular}{lc}
\hline \hline Triggered events & $3.3 \times 10^{10}$ \\
\hline L1 filtered events & $8.0 \times 10^{8}$ \\
\hline Events in final sample & 36,900 \\
\hline
\end{tabular}

described in Section 5). Downgoing muons can also be created in showers caused by gamma rays, which point back to their source like neutrinos. The possibility for IceCube to detect $\mathrm{PeV}$ gamma-ray sources in the southern sky is discussed in Halzen et al. (2009), which concludes that a realistic source could be detected using muons in the ice only after 10 years of observing. Gamma-ray sources will not be considered further here.

Two processing levels are used to reduce the approximately $3.3 \times 10^{10}$ triggered events down to a suitable sample for analysis (see Table 1). Random noise at the level of about $500 \mathrm{~Hz}$ per DOM is mainly due to radioactive decays in the materials in the DOMs. The contribution to triggered events by this random noise is highly suppressed by the local coincident hit requirement. To further reduce the contribution from noise, only hits within a $6 \mu$ s time window are used for the reconstructions. This time window is defined as the window that contains the most hits during the event. About $5 \%$ of down-going muons which trigger the detector are initially misreconstructed as up-going by the first stages of event processing. A persistent background that grows with the size of the detector is CR muons (or bundles of muons) from different showers which arrive in coincidence. At trigger level, they make up about $13 \%$ of the events. These coincident muon bundles can mimic the hit pattern of good up-going events, confusing a single-muon fit.

A likelihood-based muon track reconstruction is first performed at the South Pole (L1 filter). The likelihood function (Ahrens et al. 2004) parameterizes the probability of observing the geometry and timing of the hits in terms of a muon track's position, zenith angle, and azimuth angle. This likelihood is maximized, yielding the best-fit direction and position for the muon track. Initial fits are performed using an SPE likelihood that uses the time of the leading edge of the first photon arriving in each DOM. These reconstructions yield robust results used for the first level of background rejection. All events that are reconstructed as up-going are kept, while events in the down-going region must pass an energy cut that tightens with decreasing zenith angle. Events pass this L1 filter at an average rate of about $22 \mathrm{~Hz}$ and are buffered before transmission via a communications satellite using the South Pole Archival and Data Exchange (SPADE) system.

The processing done in the North includes a broader base of reconstructions compared to what is done at the South Pole. Rather than just the simple SPE fit, the multiple photoelectron (MPE) fit uses the number of observed photons to describe the expected arrival time of the first photon. This first photon is scattered less than an average photon when many arrive at the same DOM. The MPE likelihood description uses more available information than SPE and improves the tracking resolution as energy increases, and this reconstruction is used for the final analysis. The offline processing also provides parameters useful for background rejection, reconstructs the muon energy, and estimates the angular resolution on an eventby-event basis. Reducing the filtered events to the final sample of this analysis requires cutting on the following parameters:

1. Reduced log-likelihood. The log-likelihood from the muon track fit divided by the number of degrees of freedom, given

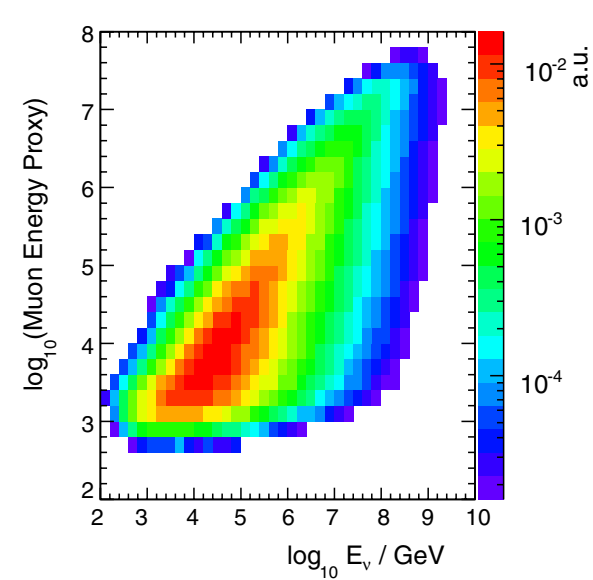

Figure 2. Distribution of the muon energy proxy (energy loss observed in the detector) vs. the true neutrino energy for a flux $d \Phi / d E \propto E^{-2}$.

by the number of DOMs with hits minus five, the number of free parameters used to describe the muon. This parameter performed poorly on low-energy signal events. It was found that low-energy efficiency could be increased by instead dividing the likelihood by number of DOMs with photon hits minus 2.5. Both the standard and modified parameters were used, requiring events to pass one selection or the other. This kept the efficiency higher for a broader energy range.

2. Angular uncertainty, $\sigma$. An estimate of the uncertainty in the muon track direction. The directional likelihood space around the best track solution is sampled and fit to a paraboloid. The contour of the paraboloid traces an error ellipse indicating how well the muon direction is localized (Neunhoffer 2006). The rms of the major and minor axes of the error ellipse is used to define a circular error. This parameter is effective both for removing misreconstructed events and as an event-by-event angular uncertainty estimator.

3. Muon energy proxy. The average photon density along the muon track, used as a proxy for the muon energy. It is calculated accounting for the distance to DOMs, their angular acceptance, and average scattering and absorption properties of photons in the ice. The energy loss of a muon moving through the detector scales with the muon energy above about $1 \mathrm{TeV}$ when stochastic energy losses due to bremsstrahlung, pair production, and photonuclear interactions dominate over ionization losses. The energy resolution obtained is of the order of 0.3 in the $\log _{10}$ of the muon energy (at closest approach to the average hit location) for energies between about $10 \mathrm{TeV}$ and $100 \mathrm{PeV}$. Since the interaction vertex is often an unknown distance from the detector, the muon in the detection volume has already lost an unknown amount of energy. Figure 2 shows the distribution of this energy parameter versus the true neutrino energy for a simulated spectrum $d \Phi / d E \propto E^{-2}$. Despite the uncertainty on the neutrino energy, for a statistical sample of events this energy estimator is a powerful analysis tool because of the wide range over which energies are measured.

4. Zenith-weighted likelihood ratio. The likelihood ratio between an unbiased muon fit and a fit with an event weight according to the known down-going muon zenith distribution as a Bayesian prior. Applied to up-going tracks, a high likelihood ratio establishes strong evidence that the event is 
actually up-going and not a misreconstructed down-going event.

5. NDir. The number of DOMs with direct photons, defined as arriving within $-15 \mathrm{~ns}$ to $+75 \mathrm{~ns}$ of the expectation from an unscattered photon emitted from the reconstructed muon track at the Cerenkov angle. Scattering of photons in the ice causes a loss of directional information and will delay them with respect to the unscattered expectation.

6. LDir. The maximum length between direct photons, projected along the best muon track solution.

7. Zenith directions of split events. The zenith angles resulting from splitting of an event into two parts and reconstructing each part separately. This is done in two ways: temporally, by using the mean photon arrival time as the split criterion, and geometrically, by using the plane both perpendicular to the track and containing the average hit location as the split criterion. This technique is effective against coincident muon bundles misreconstructed as single up-going tracks if both sub-events are required to be up-going.

In the up-going region, all parameters are used. The zenithweighted likelihood ratio and event splitting are specifically designed to remove down-going atmospheric muon backgrounds that have been misreconstructed as up-going while the other parameters focus on overall track quality.

In the down-going region, without a veto or Earth filter, muons from CR showers overwhelm neutrino-induced muons, except possibly at high energies if the neutrino source spectra are harder than the CR spectrum. The aim of the analysis in this region is therefore to select high-energy, well-reconstructed events. We use the first three parameters in the list above as cut variables, requiring a higher track quality than in the up-going range. Energy cuts were introduced in the down-going region to reduce the number of events to a suitable size, cutting to achieve a constant number of events per solid angle (which also simplifies the background estimation in the analysis). This technique keeps the high-energy events which are most important for discovery. Cuts were optimized for the best sensitivity using a simulated signal of muon neutrinos with spectrum $d \Phi / d E \propto E^{-2}$. We checked that the same cuts resulted in a nearly optimal sensitivity for a softer spectrum $d \Phi / d E \propto E^{-3}$ in the up-going region where low-energy sensitivity is possible and for a harder spectrum $d \Phi / d E \propto E^{-1.5}$ in the down-going region.

Of the 36,900 events passing all selection criteria, 14,121 are up-going events from the northern sky, mostly muons induced by atmospheric neutrinos. Simulations of CR air showers with CORSIKA (Heck et al. 1998) show a $2.4 \% \pm 0.8 \%$ contamination due to misreconstructed down-going atmospheric muons. The other 22,779 are down-going events from the southern sky, mostly high-energy atmospheric muons. An equatorial sky map of these events is given in Figure 3.

\subsection{Data and Simulation Comparison}

Simulation of neutrinos is used for determining event selection and calculating upper limits. The simulation of neutrinos is based on ANIS (Gazizov \& Kowalski 2005). Deep inelastic neutrino-nucleon cross sections use CTEQ5 parton distribution functions (Lai et al. 2000). Neutrino simulation can be weighted for different fluxes, accounting for the probability of each event to occur. In this way, the same simulation sample can be used to represent atmospheric neutrino models such as Bartol (Barr et al. 2004) and Honda (Honda et al. 2007) neutrino fluxes from pion and kaon decays (conventional flux). Neutrinos from charmed

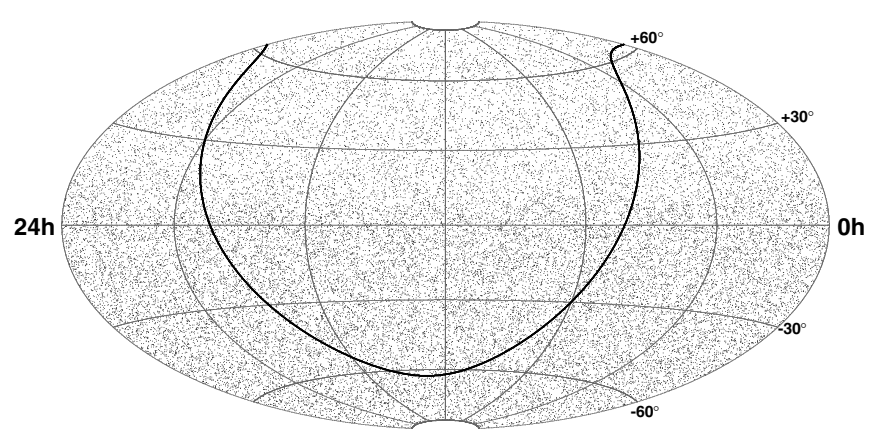

Figure 3. Equatorial skymap (J2000) of the 36,900 events in the final sample. The galactic plane is shown as the solid black curve. The northern sky (positive declinations) is dominated by up-going atmospheric neutrino-induced muons, and the southern sky (negative declinations) is dominated by muons produced in cosmic ray showers in the atmosphere above the South Pole.

meson decays (prompt flux) have been simulated according to a variety of models (Martin et al. 2003; Enberg et al. 2008; Bugaev et al. 1989). Seasonal variations in atmospheric neutrino rates are expected to be a maximum of $\pm 4 \%$ for neutrinos originating near the polar regions. Near the equator, atmospheric variations are much smaller and the variation in the number of events is expected to be less than $\pm 0.5 \%$ (Ackermann \& Bernardini 2005).

Atmospheric muon background is simulated mostly to guide and verify the event selection. Muons from CR air showers were simulated with CORSIKA (Heck et al. 1998) with the SIBYLL hadronic interaction model (Ahn et al. 2009). An October polar atmosphere, an average case over the year, is used for the CORSIKA simulation, ignoring the seasonal variations of $\pm 10 \%$ in event rates (Tilav et al. 2010). Muon propagation through the Earth and ice is done using Muon Monte Carlo (Chirkin \& Rhode 2004). Using measurements of the scattering and absorption lengths in ice (Ackermann et al. 2006), a detailed simulation propagates the photon signal to each DOM (Lundberg et al. 2007). The simulation of the DOMs includes their angular acceptance and electronics. Experimental and simulated data are processed and filtered in the same way.

In Figure 4, we show the cosine of zenith and in Figure 5 the muon energy proxy, reduced log-likelihood, and angular uncertainty estimator distributions of all events at trigger level, L1 filter level, and after final analysis cuts for data and Monte Carlo (MC). In these figures, the simulation uses a slightly modified version of the poly-gonato model of the galactic CR flux and composition (Hoerandel 2003). Above the galactic model cutoff at $Z \times 4 \times 10^{15} \mathrm{eV}$, a flux of pure iron is used with an $E^{-3}$ spectrum. This is done because currently CORSIKA cannot propagate elements in the poly-gonato model that are heavier than iron. Moreover, the poly-gonato model only accounts for galactic CRs and does not fully account for the average measured flux above $10^{17} \mathrm{eV}$, even when all nuclei are considered (see Figure 11 in Hoerandel 2003). These corrections then reproduce the measured CR spectrum at these energies. There is a $23 \%$ difference in normalization of data and CR muon events at trigger level. This normalization offset largely disappears after quality cuts are made. Generally good agreement is achieved at later cut levels.

Figure 4 shows some disagreement between data and simulation for zenith angles around $80^{\circ}$. Muons created in CR showers in the atmosphere near this zenith angle must travel about $15 \mathrm{~km}$ to reach the bottom of IceCube. Only very high energy muons can travel such distances. For the simulation to produce the 


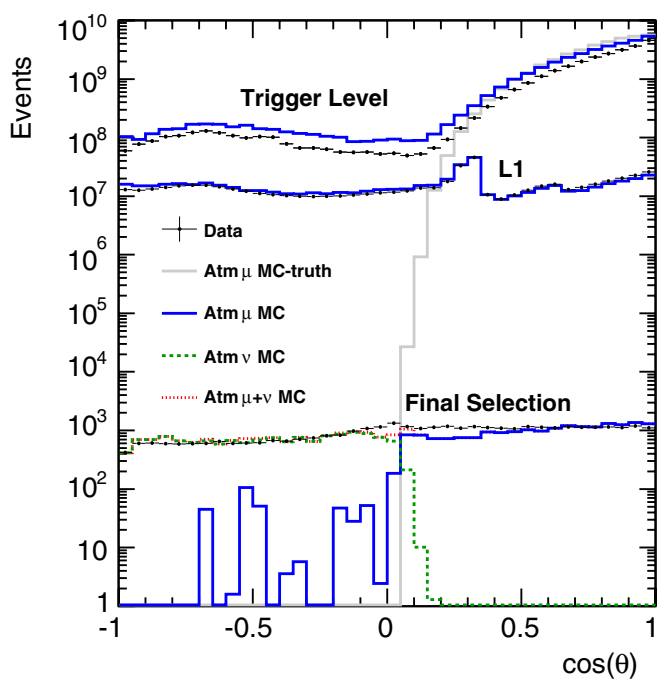

Figure 4. Distribution of reconstructed cosine zenith at trigger level, L1, and final cut level for data and simulation of atmospheric muons (Hoerandel 2003) and neutrinos (Barr et al. 2004; Bugaev et al. 1989). The true cosine zenith distribution of the muons at trigger level is also shown.

(A color version of this figure is available in the online journal.)

correct zenith distribution for these nearly horizontal events, CR composition can be important since protons can produce higher energy muons than iron nuclei with the same energy.

In addition to the slightly modified version of the poly-gonato model, discussed above, a simpler pure proton and iron twocomponent model with a much higher contribution of protons is used for comparison (Glasstetter \& Hoerandel 1999). The final zenith distribution with each of these models is shown in Figure 6 . The atmospheric muon simulation is not only affected by the primary composition uncertainties at high energy; it is also affected by the hadronic model, affecting the production rate of muons at the level of $15 \%$ in the region of interest for IceCube, greater than about $1 \mathrm{TeV}$, as discussed in the SIBYLL model paper (Ahn et al. 2009) and in the comparison between different hadronic models used in CORSIKA presented in Berghaus et al. (2008).

For the up-going region, several models of atmospheric neutrino fluxes, both conventional fluxes from pion and kaon decay and prompt fluxes from charmed meson decay, are shown in Figure 6. To represent the low and high predictions, conventional and prompt models are used in pairs: Honda (Honda et al. 2007) for the conventional flux paired with Sarcevic (Enberg et al. 2008) for the prompt flux represent the low prediction, and Bartol (Barr et al. 2004) for the conventional
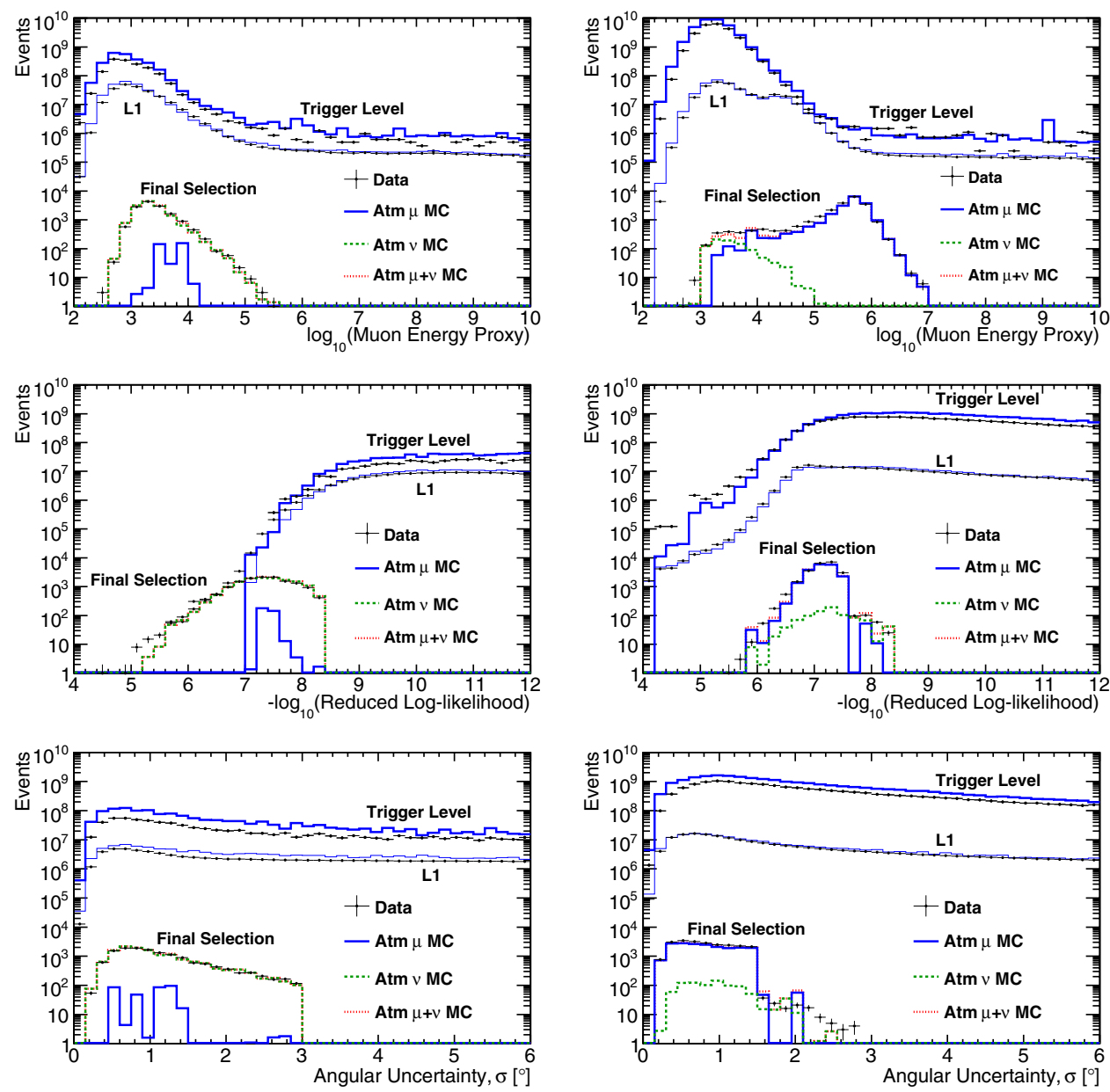

Figure 5. Distributions of muon energy proxy (top row), reduced log-likelihood (middle row), and angular uncertainty estimator (bottom row) for the up-going sample (left column) and the down-going sample (right column). Each is shown at trigger level, L1, and final cut level for data and simulation of atmospheric muons and neutrinos. In the up-going sample (left column), all atmospheric muons are misreconstructed, and at final level their remaining estimated contribution is about $2.4 \% \pm$ $0.8 \%$.

(A color version of this figure is available in the online journal.) 


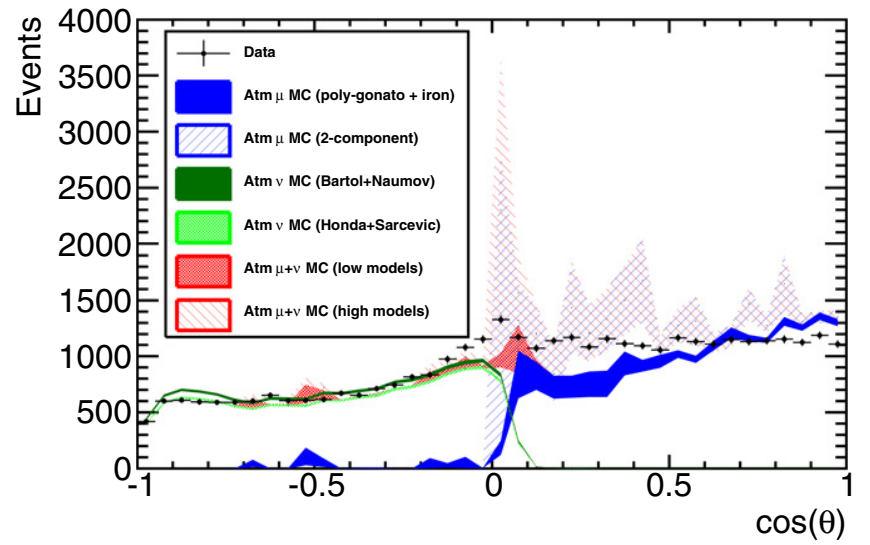

Figure 6. Distribution of reconstructed cosine zenith for the final event sample compared to the models discussed in the text. Honda and Sarcevic are summed with poly-gonato to represent the set of low predictions, and Bartol and Naumov are summed with the two-component model for the high predictions. Only statistical errors are shown. The two-component model has limited statistics, causing the peaks and valleys. Systematic uncertainties of neutrino production in CR showers are estimated to be about $40 \%$ at $1 \mathrm{TeV}$ (Barr et al. 2006) and $15 \%$ in the muon rate greater than about $1 \mathrm{TeV}$ (Ahn et al. 2009; Berghaus et al. 2008).

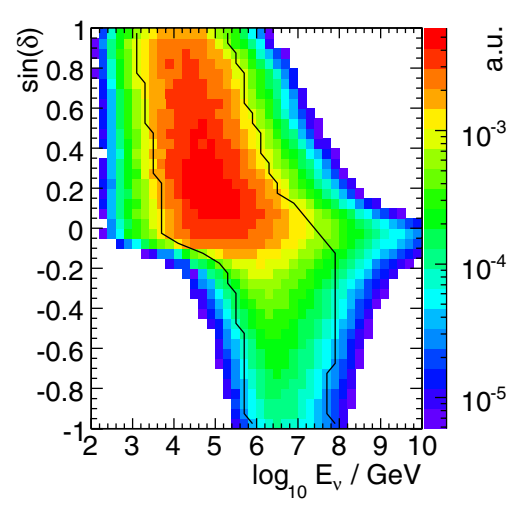

Figure 7. Energy distribution for a flux $d \Phi / d E \propto E^{-2}$ of neutrinos as a function of declination for the final event selection. The black contours indicate the $90 \%$ central containment interval for each declination.

flux paired with Naumov (Bugaev et al. 1989) for the prompt flux represent the high prediction. Additional uncertainty in the predicted atmospheric neutrino rate is estimated to be about $40 \%$ at $1 \mathrm{TeV}$ (Barr et al. 2006). We conclude that our data agree with background simulation at the final level, within the range of uncertainties allowable by existing CR composition and atmospheric neutrino models.

\section{DETECTOR PERFORMANCE}

The performance of the detector and the analysis is characterized using the simulation described in Section 3.2. For a spectrum of neutrinos $d \Phi / d E \propto E^{-2}$, the median angular difference between the neutrino and the reconstructed direction of the muon in the northern (southern) sky is $0.8(0.6)$. Along with more severe quality selection in the southern sky, the different energy distributions in each hemisphere, shown in Figure 7, cause the difference in these two values. This is because the reconstruction performs better at higher energy due to the larger amount of light and longer muon tracks. The cumulative pointspread function (PSF) is shown in Figure 8 for two energy ranges and compared with simulation of the complete IceCube detector using the same quality selection, as well as the median PSF versus energy for the two hemispheres.

The neutrino effective area $A_{v}^{\text {eff }}$ is a useful parameter to determine event rates and the performance of a detector for different analyses and fluxes. The expected event rate for a given differential flux $d \Phi / d E$ is

$$
N_{\text {events }}\left(\delta_{v}\right)=\int d E_{v} A_{v}^{\mathrm{eff}}\left(E_{v}, \delta_{v}\right) \frac{d \Phi_{\nu}\left(E_{v}, \delta_{v}\right)}{d E_{v}},
$$

and is calculable using simulation. The $A_{v}^{\text {eff }}$ represents the size of an equivalent detector if it were $100 \%$ efficient. Figure 9 shows the $A_{v}^{\text {eff }}$ for fluxes of $v_{\mu}+\bar{v}_{\mu}$ and $v_{\tau}+\bar{v}_{\tau}$, for events at final selection level. Neutrinos arriving from the highest declinations must travel through the largest column depth and can be absorbed: this accounts for the turnover at high energies for nearly vertical up-going muon neutrinos. Tau neutrinos have the advantage that the tau secondary can decay back into a tau neutrino before losing much energy.

Although tau (and electron) neutrino secondaries usually produce nearly spherical showers rather than tracks, tau leptons will decay to muons with a $17.7 \%$ branching ratio (Amsler et al. 2008). At very high energy (above about $1 \mathrm{PeV}$ ), a tau will travel far enough before decaying that the direction can be reconstructed well, contributing to any extraterrestrial signal in the muon channel. For the upper limits quoted in Section 8, we must make an assumption on the flavor ratios at Earth, after oscillations. It is common to assume $\Phi_{v_{e}}: \Phi_{v_{\mu}}: \Phi_{v_{\tau}}=1: 1: 1$. This is physically motivated by neutrino production from pion decay and the subsequent muon decay, yielding $\Phi_{v_{e}}: \Phi_{v_{\mu}}: \Phi_{\nu_{\tau}}=1: 2: 0$.
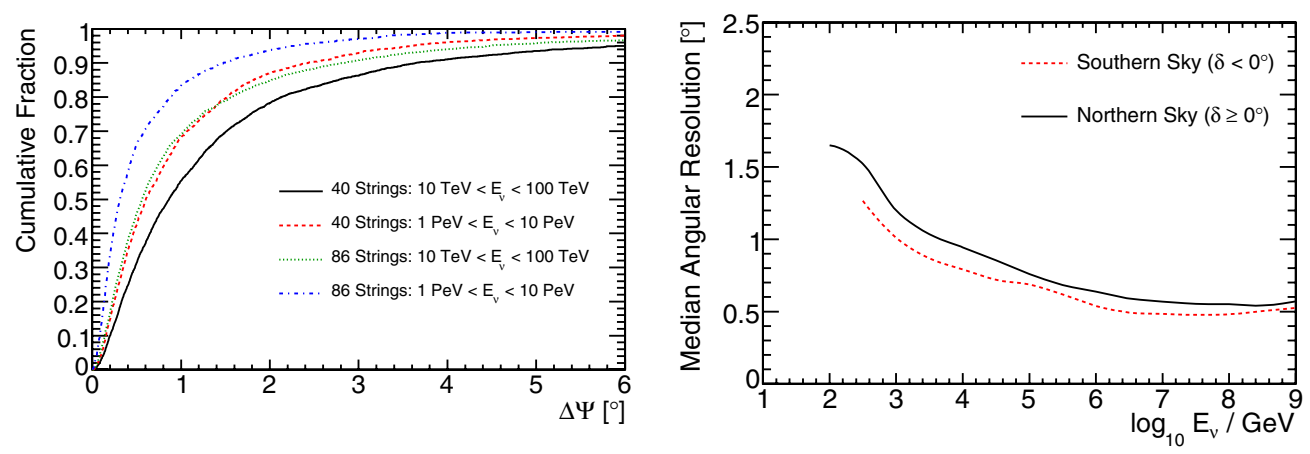

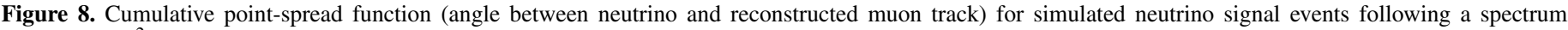

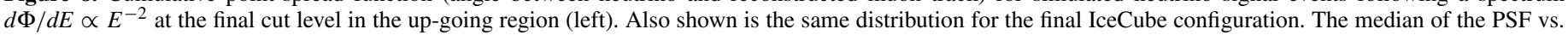
energy is shown separately for the northern and southern skies (right). The improvement in the southern sky is because of the more restrictive quality cuts. 

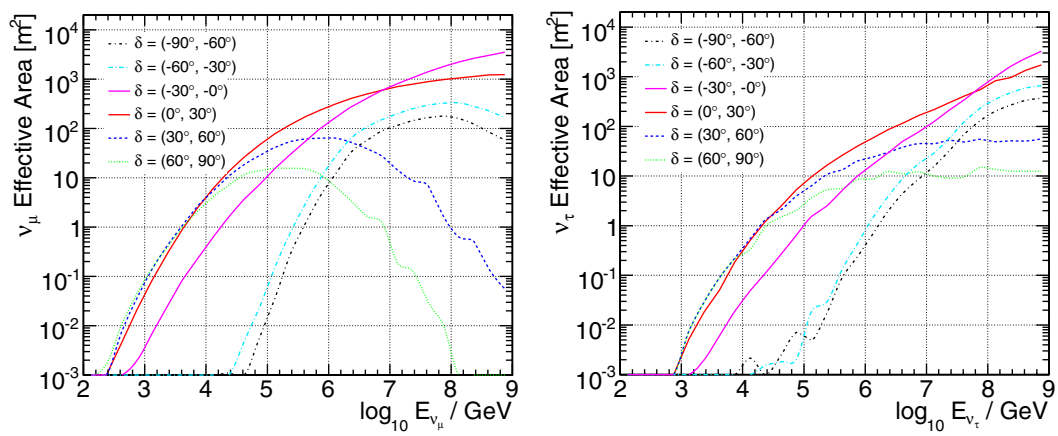

Figure 9. Solid-angle-averaged effective areas at final cut level for astrophysical neutrino fluxes in six declination bands for $v_{\mu}+\bar{v}_{\mu}($ left $)$ and $v_{\tau}+\bar{v}_{\tau}$ (right), assuming an equal flux of neutrinos and antineutrinos.

(A color version of this figure is available in the online journal.)

After standard oscillations over astrophysical baselines, this gives an equal flux of each flavor at Earth (Athar et al. 2000). Under certain astrophysical scenarios, the contribution from muon decay may be suppressed, leading to an observed flux ratio of $\Phi_{v_{e}}: \Phi_{v_{\mu}}: \Phi_{v_{\tau}}=1: 1.8: 1.8$ (Kashti \& Waxman 2005), or the contribution of tau neutrinos could be enhanced by the decay of charmed mesons at very high energy (Enberg et al. 2009). For a spectrum $d \Phi / d E \propto E^{-2}$ and equal muon and tau neutrino fluxes, the fraction of tau neutrino-induced events is about $17 \%$ for vertically down-going, $10 \%$ for horizontal, and $13 \%$ for vertically up-going. Because the contribution from tau neutrinos is relatively small, assuming only a flux of muon neutrinos can be used for convenience and to compare to other published limits. We have tabulated limits on both $\Phi_{\nu_{\mu}}$ and the sum $\Phi_{v_{\mu}}+\Phi_{v_{\tau}}$, assuming an equal flux of each, while in the figures we have specified that we only consider a flux of muon neutrinos. Limits are always reported for the flux at the surface of the Earth.

\section{SEARCH METHOD}

An unbinned maximum likelihood ratio method is used to look for a localized, statistically significant excess of events above the background. We also use energy information to help separate possible signal from the known backgrounds.

The method follows that of Braun et al. (2008). The data are modeled as a two-component mixture of signal and background. A maximum likelihood fit to the data is used to determine the relative contribution of each component. Given $N$ events in the data set, the probability density of the $i$ th event is

$$
\frac{n_{s}}{N} \mathcal{S}_{i}+\left(1-\frac{n_{s}}{N}\right) \mathcal{B}_{i}
$$

where $\mathcal{S}_{i}$ and $\mathcal{B}_{i}$ are the signal and background probability density functions (PDFs), respectively. The parameter $n_{s}$ is the unknown contribution of signal events.

For an event with reconstructed direction $\vec{x}_{i}=\left(\alpha_{i}, \delta_{i}\right)$, where $\alpha_{i}$ is the right ascension (R.A.) and $\delta_{i}$ is the declination, we model the probability of originating from the source at $\vec{x}_{s}$ as a circular two-dimensional Gaussian,

$$
\mathcal{N}\left(\vec{x}_{i} \mid \vec{x}_{s}, \sigma_{i}\right)=\frac{1}{2 \pi \sigma_{i}^{2}} \exp \left(-\frac{\left|\vec{x}_{i}-\vec{x}_{s}\right|}{2 \sigma_{i}^{2}}\right),
$$

where $\sigma_{i}$ is the angular uncertainty reconstructed for each event individually (Neunhoffer 2006) and $\left|\vec{x}_{i}-\vec{x}_{s}\right|$ is the space angle difference between the source and reconstructed event. While the average angular uncertainty decreases with

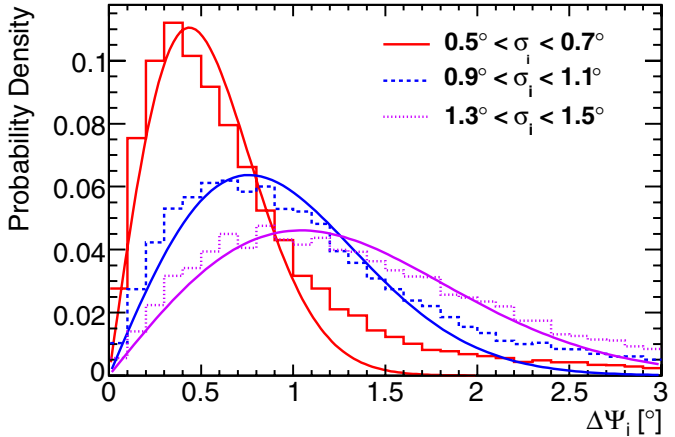

Figure 10. Angular deviation between neutrino and reconstructed muon direction $\Delta \Psi$ for ranges in $\sigma_{i}$, the reconstructed angular uncertainty estimator. Fits of these distributions to two-dimensional Gaussians projected into $\Delta \Psi$ are also shown. The value of $\sigma_{i}$ is correlated to the track reconstruction error. A small fraction of events are not well represented by the Gaussian distribution, but these are the least well-reconstructed events and contribute the least to signal detection.

(A color version of this figure is available in the online journal.)

increasing energy, the individual $\sigma_{i}$ values are estimated from the reconstruction likelihood shape itself, and therefore the PSF incorporates this dependence without explicitly being a function of energy. The PSFs for different ranges of $\sigma_{i}$ are in Figure 10, showing the correlation between the estimated angular uncertainty and actual track reconstruction error.

The energy PDF $\mathcal{E}\left(E_{i} \mid \gamma, \delta_{i}\right)$ describes the probability of obtaining a reconstructed muon energy $E_{i}$ for an event produced by a source of a given neutrino energy spectrum $E^{-\gamma}$ at declination $\delta_{i}$. We describe the energy distribution using 22 declination bands. Twenty bands, spaced evenly by solid angle, cover the down-going range where the energy distributions are changing the most due to the energy cuts in the event selection, while two are needed to sufficiently describe the upgoing events, with the separation at $\delta=15^{\circ}$. We fit the source spectrum with a power law $E^{-\gamma} ; \gamma$ is a free parameter. The probability of obtaining a reconstructed muon energy $E_{i}$ for an event produced by a source with spectral index $\gamma$, for spectral indices $1.0<\gamma<4.0$, is determined using simulation. Two examples of these energy PDFs are shown in Figure 11.

The full signal PDF is given by the product of the spatial and energy PDFs:

$$
\mathcal{S}_{i}=\mathcal{N}\left(\vec{x}_{i} \mid \vec{x}_{s}, \sigma_{i}\right) \cdot \mathcal{E}\left(E_{i} \mid \gamma, \delta_{i}\right) .
$$

The background PDF $\mathcal{B}_{i}$ contains the same terms, describing the angular and energy distributions of background events:

$$
\mathcal{B}_{i}=\mathcal{N}_{\mathrm{Atm}}\left(\vec{x}_{i}\right) \cdot \mathcal{E}\left(E_{i} \mid \operatorname{Atm}, \delta_{\mathrm{i}}\right),
$$



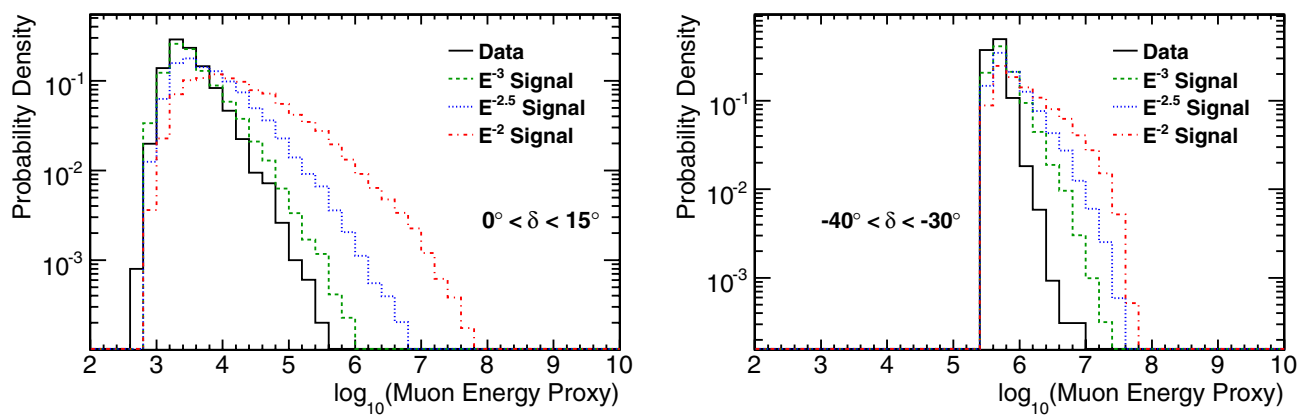

Figure 11. Probability densities for the muon energy proxy for data as well as simulated power-law neutrino spectra. Two declination bands are shown: $0^{\circ}<\delta<15^{\circ}$ (left) and $-40^{\circ}<\delta<-30^{\circ}$ (right), representing two of the declination-dependent energy PDFs used in the likelihood analysis. There is an energy cut applied for negative declinations.

(A color version of this figure is available in the online journal.)
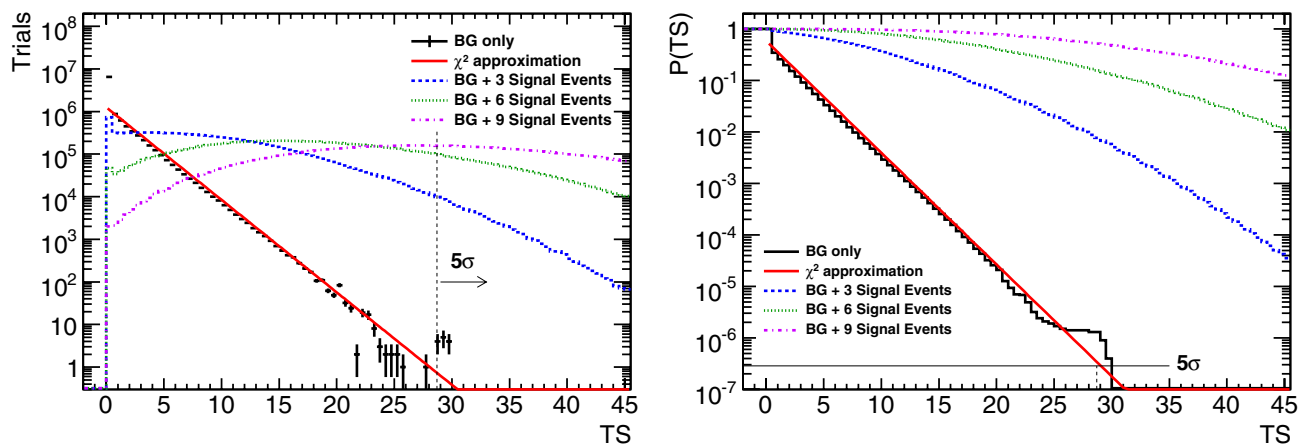

Figure 12. Distributions of the test statistic TS for a fixed point source at $\delta=25^{\circ}$ for 10 million scrambled data sets (left) and the $p$-value, or the probability to obtain TS or higher (right). A $\chi^{2}$ distribution with 2 degrees of freedom times one-half (because we only search for excesses) can be used as an approximation. Also shown are the distributions when simulated signal events are injected following a spectrum $d \Phi / d E \propto E^{-2}$. About nine events are needed for a discovery in $50 \%$ of trials at this declination since the median TS in this case is 29.1 .

(A color version of this figure is available in the online journal.)

where $\mathcal{N}_{\text {Atm }}\left(\vec{x}_{i}\right)$ is the spatial PDF of atmospheric background and $\mathcal{E}\left(E_{i} \mid \mathrm{Atm}, \delta_{i}\right)$ is the probability of obtaining $E_{i}$ from atmospheric backgrounds (neutrinos and muons) at the declination of the event. These PDFs are constructed using data and, for the energy term, in the same 22 declinations bands as the signal PDF. All non-uniformities in atmospheric background event rates caused by the detector acceptance or seasonal variation average out in the time-integrated analysis. Therefore, $\mathcal{N}_{\text {Atm }}\left(\vec{x}_{i}\right)$ has a flat expectation in R.A. and is only dependent on declination. Because the data are used in this way for background estimation, the analysis is restricted from $-85^{\circ}$ to $85^{\circ}$ declination, so that any point-source signal will still be a small contribution to the total number of events in the same declination region.

The likelihood of the data is the product of all event probability densities:

$$
\mathcal{L}\left(n_{s}, \gamma\right)=\prod_{i=1}^{N}\left[\frac{n_{s}}{N} \mathcal{S}_{i}+\left(1-\frac{n_{s}}{N}\right) \mathcal{B}_{i}\right] .
$$

The likelihood is then maximized with respect to $n_{s}$ and $\gamma$, giving the best-fit values $\hat{n}_{s}$ and $\hat{\gamma}$. The null hypothesis is given by $n_{s}=0$ ( $\gamma$ has no meaning when no signal is present). The fit has been restricted to the physical signal region $n_{s} \geqslant 0$. The likelihood ratio test statistic is

$$
\mathrm{TS}= \begin{cases}-2 \log \frac{\mathcal{L}\left(n_{s}=0\right)}{\mathcal{L}\left(\hat{n}_{s}, \hat{\gamma}_{s}\right)} & n_{s} \geqslant 0, \\ 0 & n_{s}<0 .\end{cases}
$$

The significance of the analysis is determined by comparing the TS from the real data with the distribution of TS from the null hypothesis (events scrambled in R.A.). We define the $p$-value as the fraction of randomized data sets with equal or higher test statistic values than the real data. Since we do not allow negative values of $n_{s}$, all underfluctuations result in TS $=0$, the lowest possible value. This yields a $p$-value of $100 \%$, which happens in approximately half of the searches. We evaluate the median sensitivity and upper limits at a $90 \%$ confidence level (CL) using the method of Feldman \& Cousins (1998) and calculate the discovery potential as the flux required for $50 \%$ of trials with simulated signal to yield a $p$-value less than $2.87 \times 10^{-7}$ (i.e., $5 \sigma$ significance if expressed as the one-sided tail of a Gaussian distribution). The distributions of TS and the corresponding $p$-value for 10 million trials are shown in Figure 12 for a fixed point source at $\delta=25^{\circ}$. Distributions with simulated signal events injected following a spectrum $d \Phi / d E \propto E^{-2}$ are included, as well as a $\chi^{2}$ distribution with 2 degrees of freedom, which is used to estimate the $5 \sigma$ significance threshold for calculating the discovery potential since simulating enough scrambled data sets requires a large amount of processing time.

Although sensitivities and limits for sources with $d \Phi / d E \propto$ $E^{-2}$ have become a useful benchmark for comparing performance, a wide range of other spectral indices are possible along with cutoffs over a wide range of energy. To understand the ability of the method to detect sources with cutoff spectra, typically observed in gamma rays to be in the range $1-10 \mathrm{TeV}$ for galactic sources, Figure 13 shows the discovery potentials for a wide range of exponential cutoffs, demonstrating the ability of the method to detect sources with cutoff spectra. Typically, cutoffs observed in gamma rays are in the range $1-10 \mathrm{TeV}$ for galactic 

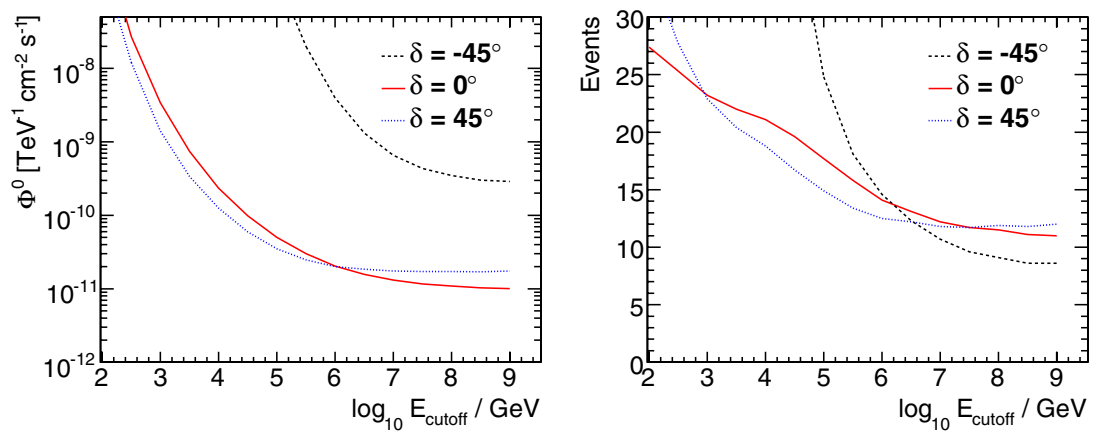

Figure 13. Discovery potential is shown as a function of the cutoff energy for a flux parameterized as $d \Phi / d E=\Phi^{0} \cdot(E / \mathrm{TeV})^{-2} \exp \left(-E / E_{\text {cutoff }}\right)$. The discovery potential is given as the flux normalization $\Phi^{0}$ (left) and the number of events at the final level (right). Curves are shown at three representative declinations. The likelihood fit is still performed using a pure power law.

(A color version of this figure is available in the online journal.)

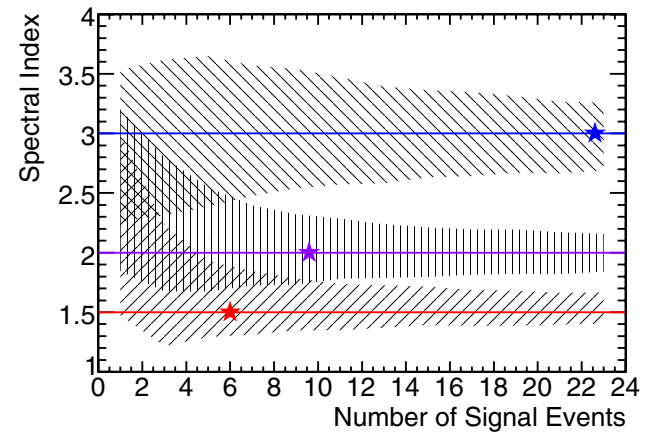

Figure 14. Reconstructed spectral index ( $1 \sigma$ shaded area) vs. the number of signal events injected for three source spectra: $E^{-1.5}, E^{-2}$, and $E^{-3}$. The sources are pure power laws at a declination of $6^{\circ}$. The stars mark the average number of events required for a $5 \sigma$ discovery for each spectrum. Systematic errors are not included.

(A color version of this figure is available in the online journal.)

sources. The likelihood fit is still performed using a pure power law.

The likelihood analysis is not only more sensitive than binned methods, but it can also help extract astrophysical information. Figure 14 shows our ability to reconstruct the spectral index for power-law neutrino sources at a declination of $6^{\circ}$. The effective area is high for a broad range of energies here, and the spectral resolution is best. For each spectrum shown, the statistical uncertainty $(1 \sigma \mathrm{CL})$ in the spectral index will be about \pm 0.3 when enough events are present to claim a discovery. Spectral resolution worsens to \pm 0.4 at both $\delta=-45^{\circ}$ and $\delta=45^{\circ}$ when enough events are present for a discovery in each case.

Stacking multiple sources in neutrino astronomy has been an effective way to enhance discovery potential and further constrain astrophysical models (Achterberg et al. 2006b; Abbasi et al. 2009c). We can consider the accumulated signal from a collection of sources using a method similar to Abbasi et al. (2006). Only a modification to the signal likelihood is necessary in order to stack sources, breaking the signal hypothesis into the sum over $M$ sources:

$$
\mathcal{S}_{i} \Rightarrow \mathcal{S}_{i}^{\mathrm{tot}}=\frac{\sum_{j=1}^{M} W^{j} R^{j}(\gamma) \mathcal{S}_{i}^{j}}{\sum_{j=1}^{M} W^{j} R^{j}(\gamma)},
$$

where $W^{j}$ is the relative theoretical weight, $R^{j}(\gamma)$ is the relative detector acceptance for a source with spectral index $\gamma$ (assumed to be the same for all stacked sources), and $\mathcal{S}_{i}^{j}$ is the signal

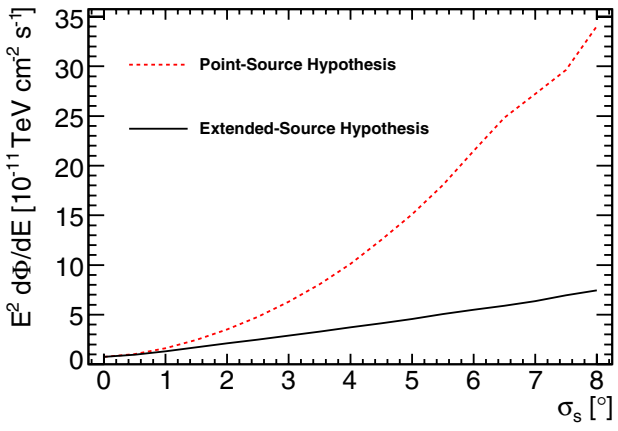

Figure 15. Discovery potential flux vs. the $\sigma_{s}$ of an extended source (distributed as a two-dimensional Gaussian) with a spectrum $d \Phi / d E \propto E^{-2}$ at a declination of $25^{\circ}$. The case of a point-source hypothesis is compared against the correct extended-source hypothesis matching what was used to simulate the signal.

(A color version of this figure is available in the online journal.)

probability density for the $i$ th event, all for the $j$ th source. As before, the total signal events $n_{s}$ and collective spectral index $\gamma$ are fit parameters. The $W^{j}$ coefficients depend on our prior theoretical assumptions about the expected neutrino luminosity. They are higher for sources that are, on theoretical grounds, expected to be brighter. Tables for $R^{j}(\gamma)$, given as the mean number of events from a source with $d \Phi / d E \propto E^{-\gamma}$, are calculated using simulation. The flexibility built into the method by the relative detector acceptance and theoretical weights allows us to use source catalogs covering the whole sky and with large variations in source strengths, as well as to directly test model predictions.

We would also like to consider sources that are spatially extended (with respect to the PSF). For an example of how important this can be, the significance observed by the Milagro experiment in the location of the Fermi source J0634.0+1745 (associated with the Geminga pulsar) rises from $3.5 \sigma$ to $6.3 \sigma$ by fitting for an extended source (Abdo et al. 2009). The only modification to the method required is to convolve the source distribution with the PSF. Since we model our PSF as a circular two-dimensional Gaussian distribution, it is easy to also model a source as a circular two-dimensional Gaussian of width $\sigma_{\mathrm{s}}$. The convolution results in a broader two-dimensional Gaussian of width $\sqrt{\sigma_{i}^{2}+\sigma_{\mathrm{s}}^{2}}$ and the likelihood uses this distribution for the signal spatial term. The discovery potential flux for a range of source extensions is shown in Figure 15 and compared to the (incorrect) hypothesis of a point source. For a source with true extent $\sigma_{s}=2^{\circ}$, the point-source hypothesis requires nearly 
a factor of two times more flux for discovery compared to the correct extended-source hypothesis.

\section{DESCRIPTION OF THE FIVE SEARCHES}

We have performed five searches:

1. a scan for the most significant point source in the entire sky;

2. a search over an a priori defined list of 39 interesting astrophysical objects;

3. a stacking search for 16 Milagro TeV gamma-ray sources, some seen only in coincidence with the Fermi-LAT, and one unconfirmed hot spot (17 total sources);

4. a stacking search for 127 local starburst galaxies (Becker et al. 2009);

5. a stacking search for five nearby clusters of galaxies (CGs), testing four different models for the CR spatial distribution (Murase et al. 2008).

The analyses and event selection procedure were determined before unblinding the R.A. of the data. We require a $5 \sigma$ significance for discovery. Final $p$-values are calculated for each search individually.

\subsection{All-sky Scan}

The first search is a scan for the single most significant point source of neutrinos over the declination range $-85^{\circ}$ to $+85^{\circ}$. The maximum likelihood ratio is defined continuously over the sky, and we sample it on a grid of $0.1 \mathrm{in} \mathrm{R.A.} \mathrm{and} 0.1 \mathrm{in} \mathrm{decl.} \mathrm{The} \mathrm{size}$ of the grid is not important as long as it is small compared to the angular resolution of the detector. Using a finer grid increases the computation time with no added benefit. A grid size that is comparable to or larger than the angular resolution could miss the location of the peaks in the significance map, yielding sub-optimal performance.

\subsection{A Priori Source List}

In order to avoid the large number of effective trials associated with scanning the entire sky, we also perform a search for the most significant of 39 a priori selected source candidates, given in Table 3. These sources have been selected on the basis of observations in gamma rays or astrophysical modeling that predicts neutrino emission. We also added the most significant location observed in the 22-string IceCube configuration (a posttrial $p$-value of 1.3\%; Abbasi et al. 2009b).

\subsection{Milagro TeV Source Stacking}

The Milagro Collaboration has reported 16 sources of $\mathrm{TeV}$ gamma rays (Abdo et al. 2007), several only after correlating with GeV gamma rays from the Fermi Gamma-ray Space Telescope source list (Abdo et al. 2009). These sources are promising candidates for detection by neutrino telescopes. Particularly interesting are sources in the complex Cygnus region (Beacom \& Kistler 2007) and six SNR associations (Halzen et al. 2008; Gonzalez-Garcia et al. 2009), including MGRO J1852+01, a hot spot that falls below the significance threshold of the Milagro Collaboration to be claimed as a source. If confirmed as a source, MGRO J1852+01 could contribute a large fraction (about 42\%) of the total neutrino flux from the SNR sources (Halzen et al. 2008). For the 40-string configuration of IceCube, the model of Halzen et al. (2008) predicts 3.0 neutrino events in 375.5 days, following a spectrum $d \Phi / d E \propto E^{-2.1}$ with an exponential cutoff at about $600 \mathrm{TeV}$.
We performed a stacking search for 17 sources observed in $\mathrm{TeV}$ gamma rays by Milagro (adding MGRO J1852+01 to the 16 sources which were found significant by the Milagro Collaboration) using an equal weight for each source in the likelihood. Assuming that neutrino and gamma-ray fluxes correlate and using these as weights in the likelihood did not appreciably improve the sensitivity in this case. Spatial extensions were used in the search for three of the sources where measurements were given (also used in the source simulation for limit calculations). The largest source was MGRO J2031+41, reported to have a diameter of $3.0 \pm 0.9$ (Abdo et al. 2007).

\subsection{Starburst Galaxy Stacking}

Starburst galaxies have a dense interstellar medium and high star formation rates, particularly of high-mass stars. This leads to both high supernova rates and heating of ambient dust. The model of Becker et al. (2009) associates the far-infrared (FIR) emission with this hot dust and the radio emission with synchrotron losses of CR electrons, presumably accelerated along with hadronic CRs in the elevated number of SNRs. The observed strong correlation between the FIR and radio emission points to the high star formation rate as the single underlying cause, and should also correlate with the neutrino flux. The increased production of CRs and high density of target material are ideal conditions for neutrino production. The starburst galaxies M82 and NGC 253 have been observed in gamma rays at $\mathrm{GeV}-\mathrm{TeV}$ energies (Abdo et al. 2010; Acciari et al. 2009; Aharonian et al. 2005) and are the only observed steady extragalactic $\mathrm{TeV}$ gamma-ray sources not associated with AGNs.

We performed a stacking search for 127 starburst galaxies, weighting the sources by their observed FIR flux at $60 \mu \mathrm{m}$, as compiled in Table A.1 in Becker et al. (2009).

\subsection{Galaxy Cluster Stacking}

CGs are another potential source of high-energy protons and, through interactions with intracluster material (ICM), neutrinos. CGs are the largest gravitationally bound objects in the universe and continue to grow through merging and accretion of dark matter and baryonic gas, generating shock fronts on megaparsec scales. The possibility for CGs to be sources of ultra-high-energy CRs above $3 \times 10^{18} \mathrm{eV}$ is described in, e.g., Norman et al. (1995) and Kang et al. (1997). Murase et al. (2008) discuss the possibility of CGs being a significant contribution to the $\mathrm{CR}$ spectrum between the second knee at about $3 \times 10^{17} \mathrm{eV}$ and the ankle at about $3 \times 10^{18} \mathrm{eV}$. They give predictions for neutrinos from five nearby $(z<$ 0.03) CGs: Virgo, Centaurus, Perseus, Coma, and Ophiuchus. Information on location, distance, and size of CGs (virial radii) was taken from Reiprich \& Boehringer (2002). These nearby CGs appear to us as spatially extended objects with virial radii subtending 1.3-6.9, so an extended spatial distribution of neutrinos is possible. Whereas the distribution of the ICM is well known from X-ray observations (Pfrommer \& Ensslin 2004), the distribution of CRs is highly uncertain. The distribution of neutrinos is given by the product of the CR and ICM distributions. Four CR models have been considered for neutrino production, discussed in Murase et al. (2008) and references therein (e.g., Colafrancesco \& Blasi 1998; Berezinsky et al. 1997):

1. Model A. CRs are uniformly distributed within the cluster shock radius, taken to be 0.56 of the virial radius for the dynamical parameters considered. 
Table 2

Galaxy Cluster Parameters

\begin{tabular}{lcccccccc}
\hline \hline Source & R.A. $\left(^{\circ}\right)$ & Decl. $\left(^{\circ}\right)$ & Model & $\sigma_{s}\left({ }^{\circ}\right)$ & $A\left(\mathrm{TeV}^{-1} \mathrm{~cm}^{-2} \mathrm{~s}^{-1}\right)$ & $\gamma_{1}$ & $\gamma_{2}$ & $E_{\text {break }}(\mathrm{TeV})$ \\
\hline Virgo & \multirow{2}{*}{186.63} & 12.72 & Model A & 2.0 & $1.42 \times 10^{-12}$ & -2.14 & -4.03 & $2.16 \times 10^{6}$ \\
& & & Model B & 4.0 & $1.18 \times 10^{-12}$ & -2.14 & -4.03 & $2.16 \times 10^{6}$ \\
& & & Isobaric & 3.0 & $7.57 \times 10^{-13}$ & -2.14 & -4.03 & $2.16 \times 10^{6}$ \\
& & & Central AGN & 0.0 & $6.47 \times 10^{-12}$ & -2.42 & -4.24 & $2.13 \times 10^{6}$ \\
\hline Centaurus & \multirow{2}{*}{192.20} & \multirow{2}{*}{41.31} & Model A & 0.25 & $2.78 \times 10^{-13}$ & -2.14 & -4.03 & $2.15 \times 10^{6}$ \\
& & & Model B & 0.5 & $2.20 \times 10^{-13}$ & -2.14 & -4.03 & $2.15 \times 10^{6}$ \\
& & & Isobaric & 0.25 & $1.09 \times 10^{-13}$ & -2.15 & -4.07 & $2.33 \times 10^{6}$ \\
& & & Central AGN & 0.0 & $5.10 \times 10^{-13}$ & -2.45 & -4.28 & $2.39 \times 10^{6}$ \\
\hline Perseus & \multirow{2}{*}{49.95} & \multirow{2}{*}{41.52} & Model A & 0.0 & $5.83 \times 10^{-14}$ & -2.15 & -4.07 & $2.32 \times 10^{6}$ \\
& & & Model B & 0.5 & $4.60 \times 10^{-14}$ & -2.15 & -4.07 & $2.32 \times 10^{6}$ \\
& & & Isobaric & 0.0 & $6.17 \times 10^{-13}$ & -2.15 & -4.07 & $2.32 \times 10^{6}$ \\
& & & Central AGN & 0.0 & $5.97 \times 10^{-13}$ & -2.40 & -4.20 & $1.88 \times 10^{6}$ \\
\hline Coma & \multirow{2}{*}{194.95} & \multirow{2}{*}{27.94} & Model A & 0.25 & $2.14 \times 10^{-14}$ & -2.14 & -4.03 & $2.12 \times 10^{6}$ \\
& & & Model B & 0.25 & $1.34 \times 10^{-14}$ & -2.14 & -4.03 & $2.12 \times 10^{6}$ \\
& & & Isobaric & 0.25 & $1.83 \times 10^{-13}$ & -2.15 & -4.07 & $2.30 \times 10^{6}$ \\
& & & Central AGN & 0.0 & $2.13 \times 10^{-13}$ & -2.41 & -4.20 & $1.89 \times 10^{6}$ \\
\hline \multirow{2}{*}{ Ophiuchus } & \multirow{2}{*}{258.11} & \multirow{2}{*}{23.36} & Model A & 0.0 & $4.87 \times 10^{-14}$ & -2.15 & -4.07 & $2.29 \times 10^{6}$ \\
& & & Model B & 0.5 & $1.50 \times 10^{-14}$ & -2.15 & -4.07 & $2.29 \times 10^{6}$ \\
& & & Isobaric & 0.0 & $5.50 \times 10^{-13}$ & -2.15 & -4.11 & $2.49 \times 10^{6}$ \\
& & & Central AGN & 0.0 & $2.55 \times 10^{-13}$ & -2.43 & -4.24 & $2.12 \times 10^{6}$ \\
\hline
\end{tabular}

Notes. $\sigma_{s}$ is the optimized sigma of a two-dimensional Gaussian distribution used in the likelihood. Numerical calculations of the differential fluxes (K. Murase 2009, private communication) for each model described in Murase et al. (2008) are fit well to broken power laws, parameterized in Equation (9).

2. Model B. CRs are uniformly distributed within the virial radius, yielding the most conservative neutrino flux distributed over the largest area.

3. Isobaric. CRs follow the distribution of thermal gas.

4. Central AGN. In a two-step acceleration scenario, CRs are accelerated in the central AGN up to a maximum energy before diffusing throughout the cluster and possibly undergoing further acceleration. For the purposes of IceCube searches, this model can be treated as a point source. This model is discussed in detail by Kotera et al. (2009).

Signal neutrinos were simulated according to each of the four models. We modeled the source extensions in the likelihood as two-dimensional Gaussian distributions with the width for each source and each model determined by optimizing for the best discovery potential. Although the modeling of the source extension as a Gaussian in the likelihood is not ideal, it is straightforward and computationally fast. The exact shape of the sources is not important for small signals; we may be able to analyze the shape with more detail depending on the intensity of any signal.

We performed a stacking search for five nearby CGs mentioned above following the model predictions of Murase et al. (2008) as weights in the likelihood. The size of the clusters in the likelihood fit was allowed to vary discretely between the optimal widths for each CR distribution model. The optimal width and $v_{\mu}$ differential flux for each source and each model are given in Table 2. The differential fluxes are parameterized as broken power laws:

$$
\frac{d \Phi}{d E}\left(\mathrm{TeV}^{-1} \mathrm{~cm}^{-2} \mathrm{~s}^{-1}\right)= \begin{cases}A \cdot(E / \mathrm{TeV})^{-\gamma_{1}} & E \leqslant E_{\text {break }} \\ B \cdot(E / \mathrm{TeV})^{-\gamma_{2}} & E>E_{\text {break }}\end{cases}
$$

The parameter $B=A \cdot E_{\mathrm{break}}^{\gamma_{2}-\gamma_{1}}$ after enforcing continuity at the break energy.

\section{SYSTEMATIC ERRORS}

The analyses described in Section 6 give reliable statistical results ( $p$-values) due to the ability to generate backgroundonly data sets by scrambling the data in R.A. By using the data to estimate background, the systematic errors come only from signal and detector simulation used to calculate flux upper limits, sensitivities, and discovery potentials.

The main systematic uncertainties on the flux limits come from photon propagation in ice, absolute DOM sensitivity, and uncertainties in the Earth density profile as well as muon energy loss. All numbers are for a spectrum $d \Phi / d E \propto E^{-2}$ of muon neutrinos. We evaluate the systematic uncertainty due to photon propagation by performing dedicated signal simulations with scattering and absorption coefficients varied within their uncertainties of $\pm 10 \%$ (Ackermann et al. 2006). The maximum difference was between the case where both scattering and absorption were increased by $10 \%$ and the case where both were decreased by $10 \%$. The deviation in the observed number of events between these two cases was $11 \%$. The range of uncertainty in the DOM sensitivity is taken as $\pm 8 \%$, based on the measured uncertainty in the PMT sensitivity (Abbasi et al. 2010a). Similarly, another dedicated simulation where we varied the DOM sensitivity inside the uncertainty leads to a maximum systematic uncertainty on the number of events of $9 \%$. These uncertainties on the flux varied by only about $2 \%$ between the northern and southern sky, so only averages over the whole sky are reported. Finally, uncertainties in muon energy losses, the neutrino-nucleon cross section, and the rock density near the detector introduce an $8 \%$ systematic uncertainty for vertically up-going events (Achterberg et al. 2007). These events are the most affected, and this uncertainty is applied to all zeniths to be conservative. A sum in quadrature of the systematic uncertainties on the flux gives a total of $16 \%$ systematic uncertainty in the signal simulation. These 
Table 3

Results for the A Priori Source Candidate List

\begin{tabular}{|c|c|c|c|c|c|c|c|c|c|}
\hline Object & R.A. $\left({ }^{\circ}\right)$ & Decl. $\left({ }^{\circ}\right)$ & $\Phi_{v_{\mu}}^{90}$ & $\Phi_{v_{\mu}+v_{\tau}}^{90}$ & $p$-value & $n_{s}$ & $\gamma$ & $N_{1^{\circ}}$ & $B_{1^{\circ}}^{\circ}$ \\
\hline Cyg OB2 & 308.08 & 41.51 & 6.04 & 10.54 & 1.00 & 0.0 & $\ldots$ & 2 & 1.8 \\
\hline MGRO J2019+37 & 305.22 & 36.83 & 7.50 & 13.3 & 0.44 & 1.0 & 2.8 & 2 & 1.9 \\
\hline MGRO J1908+06 & 286.98 & 6.27 & 3.73 & 6.82 & 0.43 & 1.5 & 3.9 & 4 & 3.1 \\
\hline Cas A & 350.85 & 58.81 & 9.04 & 15.92 & 1.00 & 0.0 & $\ldots$ & 1 & 1.8 \\
\hline IC443 & 94.18 & 22.53 & 3.80 & 6.62 & 1.00 & 0.0 & $\ldots$ & 1 & 2.0 \\
\hline Geminga & 98.48 & 17.77 & 3.91 & 6.66 & 0.48 & 0.7 & 2.1 & 1 & 2.3 \\
\hline Crab Nebula & 83.63 & 22.01 & 3.70 & 6.58 & 1.00 & 0.0 & $\ldots$ & 1 & 2.0 \\
\hline 1ES $1959+650$ & 300.00 & 65.15 & 10.74 & 19.18 & 1.00 & 0.0 & $\ldots$ & 0 & 2.0 \\
\hline 1ES $2344+514$ & 356.77 & 51.70 & 7.24 & 12.96 & 1.00 & 0.0 & $\ldots$ & 0 & 1.8 \\
\hline $3 \mathrm{C} 66 \mathrm{~A}$ & 35.67 & 43.04 & 10.89 & 19.70 & 0.24 & 3.4 & 3.9 & 3 & 1.9 \\
\hline H $1426+428$ & 217.14 & 42.67 & 6.14 & 10.94 & 1.00 & 0.0 & $\ldots$ & 3 & 1.8 \\
\hline BL Lac & 330.68 & 42.28 & 10.80 & 18.70 & 0.25 & 2.6 & 3.9 & 3 & 1.8 \\
\hline Mrk 501 & 253.47 & 39.76 & 8.11 & 14.14 & 0.41 & 1.3 & 3.9 & 3 & 2.0 \\
\hline Mrk 421 & 166.11 & 38.21 & 11.71 & 20.14 & 0.15 & 2.6 & 1.9 & 2 & 2.0 \\
\hline W Comae & 185.38 & 28.23 & 4.46 & 8.06 & 1.00 & 0.0 & $\ldots$ & 0 & 1.9 \\
\hline 1ES $0229+200$ & 38.20 & 20.29 & 6.89 & 12.06 & 0.19 & 4.0 & 2.8 & 4 & 2.1 \\
\hline M87 & 187.71 & 12.39 & 3.42 & 5.98 & 1.00 & 0.0 & $\ldots$ & 2 & 2.5 \\
\hline S5 $0716+71$ & 110.47 & 71.34 & 13.28 & 23.56 & 1.00 & 0.0 & $\ldots$ & 0 & 1.6 \\
\hline M82 & 148.97 & 69.68 & 19.14 & 32.84 & 0.40 & 2.0 & 3.9 & 4 & 1.8 \\
\hline $3 \mathrm{C} 123.0$ & 69.27 & 29.67 & 5.59 & 10.66 & 0.44 & 1.3 & 2.7 & 1 & 1.9 \\
\hline $3 C 454.3$ & 343.49 & 16.15 & 3.42 & 5.92 & 1.00 & 0.0 & $\ldots$ & 1 & 2.3 \\
\hline 4C 38.41 & 248.81 & 38.13 & 6.77 & 11.86 & 0.48 & 0.9 & 3.9 & 2 & 2.0 \\
\hline PKS $0235+164$ & 39.66 & 16.62 & 6.77 & 11.62 & 0.15 & 5.3 & 3.0 & 5 & 2.3 \\
\hline PKS 0528+134 & 82.73 & 13.53 & 3.63 & 6.72 & 1.00 & 0.0 & $\ldots$ & 2 & 2.4 \\
\hline PKS $1502+106$ & 226.10 & 10.49 & 3.26 & 5.78 & 1.00 & 0.0 & $\ldots$ & 0 & 2.5 \\
\hline $3 C 273$ & 187.28 & 2.05 & 3.61 & 6.54 & 1.00 & 0.0 & $\ldots$ & 3 & 3.4 \\
\hline NGC 1275 & 49.95 & 41.51 & 6.04 & 10.54 & 1.00 & 0.0 & $\ldots$ & 2 & 1.8 \\
\hline Cyg A & 299.87 & 40.73 & 7.84 & 13.44 & 0.46 & 1.0 & 3.5 & 3 & 1.9 \\
\hline IC-22 maximum & 153.38 & 11.38 & 3.26 & 5.86 & 1.00 & 0.0 & $\ldots$ & 1 & 2.5 \\
\hline Sgr A* & 266.42 & -29.01 & 80.56 & 139.26 & 0.41 & 1.1 & 2.7 & 4 & 3.3 \\
\hline PKS 0537-441 & 84.71 & -44.09 & 113.90 & 201.82 & 1.00 & 0.0 & $\ldots$ & 3 & 3.5 \\
\hline Cen A & 201.37 & -43.02 & 109.51 & 191.56 & 1.00 & 0.0 & $\ldots$ & 4 & 3.5 \\
\hline PKS 1454-354 & 224.36 & -35.65 & 92.56 & 156.74 & 1.00 & 0.0 & $\ldots$ & 4 & 3.5 \\
\hline PKS 2155-304 & 329.72 & -30.23 & 105.41 & 182.90 & 0.28 & 1.7 & 3.9 & 3 & 3.4 \\
\hline PKS 1622-297 & 246.53 & -29.86 & 152.28 & 263.86 & 0.048 & 3.0 & 2.6 & 4 & 3.3 \\
\hline QSO 1730-130 & 263.26 & -13.08 & 24.83 & 43.30 & 1.00 & 0.0 & $\ldots$ & 4 & 3.5 \\
\hline PKS 1406-076 & 212.24 & -7.87 & 16.04 & 28.72 & 0.42 & 1.3 & 2.3 & 4 & 3.3 \\
\hline QSO 2022-077 & 306.42 & -7.64 & 12.18 & 21.78 & 1.00 & 0.0 & $\ldots$ & 2 & 3.3 \\
\hline $3 \mathrm{C} 279$ & 194.05 & -5.79 & 11.94 & 21.36 & 0.33 & 3.6 & 3.0 & 7 & 3.5 \\
\hline
\end{tabular}

Notes. $\Phi_{v_{\mu}}^{90}$ and $\Phi_{v_{\mu}+v_{\tau}}^{90}$ are the upper limits of the Feldman-Cousins $90 \%$ confidence intervals for a $d \Phi / d E \propto E^{-2}$ flux normalization of $v_{\mu}$ and $v_{\mu}+v_{\tau}$, respectively, in units of $10^{-12} \mathrm{TeV}^{-1} \mathrm{~cm}^{-2} \mathrm{~s}^{-1}$ (i.e., $d \Phi / d E \leqslant \Phi^{90} \cdot(E / \mathrm{TeV})^{-2}$ ). $n_{s}$ is the best-fit number of signal events; the (pre-trial) $p$-value is also calculated and the spectral index $\gamma$ is given when $n_{s}>0 . N_{1^{\circ}}$ is the actual number of events observed in a bin of radius $1^{\circ}$. The background event density at the source declination is indicated by the mean number of background events $B_{1}$ expected in a bin of radius $1^{\circ}$.

systematic uncertainties are incorporated into the upper limit and sensitivity calculations using the method of Conrad et al. (2003) with a modification by Hill (2003).

\section{RESULTS}

The results of the all-sky scan are shown in the map of the pretrial $p$-values in Figure 16. The most significant deviation from background is located at 113.75 R.A., $15.15 \mathrm{decl}$. The bestfit parameters are $n_{s}=11.0$ signal events above background, with spectral index $\gamma=2$. 1 . Since the best-fit spectral index is substantially less than the expectation from background, much of the significance comes from the higher energies of the associated events. The pre-trial estimated $p$-value of the maximum log-likelihood ratio at this location is $5.2 \times 10^{-6}$. In trials using data sets scrambled in R.A., 1817 out of 10,000 have an equal or higher significance somewhere in the sky, resulting in the post-trial $p$-value of $18 \%$. Upper limits for a flux $d \Phi / d E \propto E^{-2}$ of $v_{\mu}+\bar{v}_{\mu}$ are presented in Figure 17. In all cases, an equal flux of neutrinos and antineutrinos is assumed.

The results of the point-source search in the direction of 39 source candidates selected a priori are given in Table 3 and also shown in Figure 18 with the IceCube median sensitivity. Since the fit was restricted to physical signal values $n_{s} \geqslant$ 0 , approximately half of the results have $n_{s}=0$ exactly, corresponding to $p$-values equal to $100 \%$ and upper limits equal to the median upper limit (i.e., the sensitivity). The most significant source on the list was PKS 1622-297 with a pretrial estimated $p$-value of $5 \%$. The post-trial $p$-value of $62 \%$ was again determined as the fraction of scrambled data sets with at least one source with an equal or higher significance. The mean number of events at the final cut level required for the discovery of a point source is also shown in Figure 19, along with the average background in a circular bin with $1^{\circ}$ radius. Included 


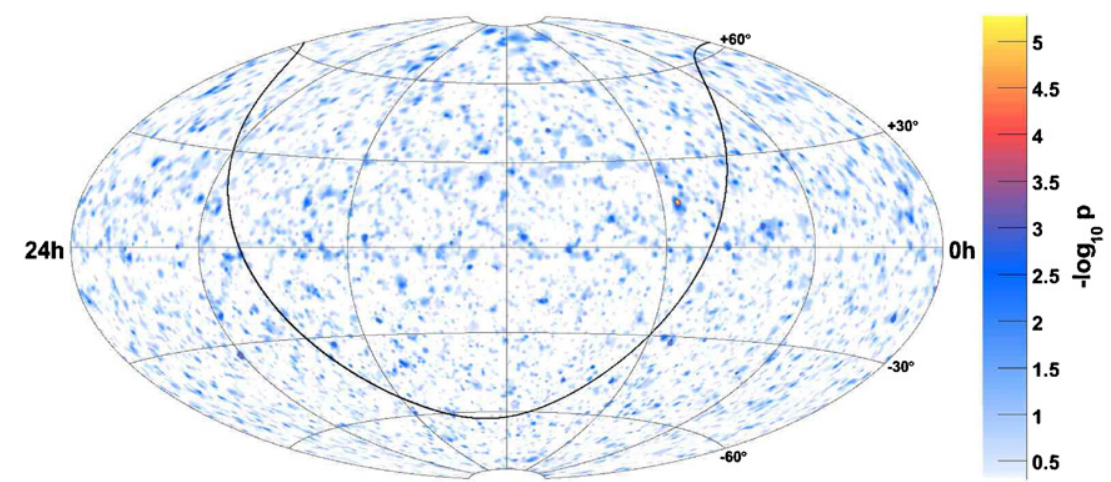

Figure 16. Equatorial skymap (J2000) of pre-trial significances ( $p$-value) of the all-sky point-source scan. The galactic plane is shown as the solid black curve.

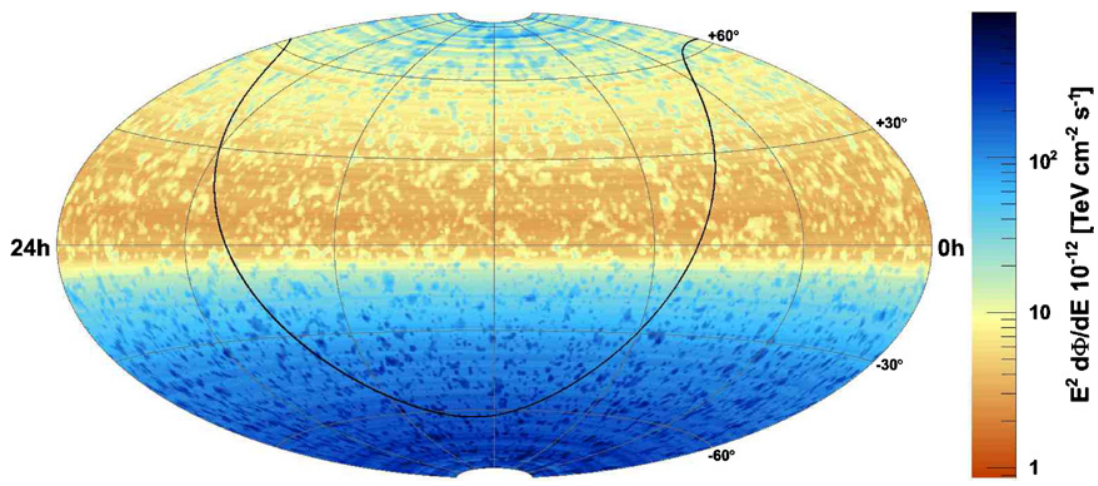

Figure 17. Equatorial skymap (J2000) of upper limits of Feldman-Cousins $90 \%$ confidence intervals for a flux $\Phi / d E \propto E^{-2}$ of $v_{\mu}+\bar{v}_{\mu}$. The galactic plane is shown as the solid black curve.

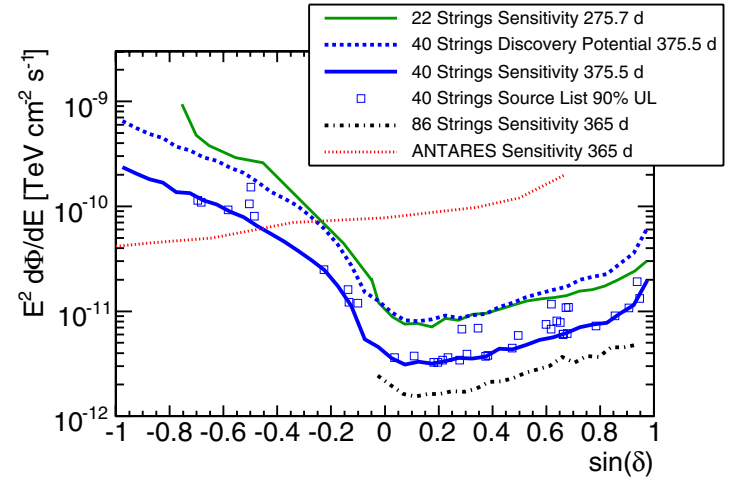

Figure 18. Median sensitivity to a point-source flux $d \Phi / d E \propto E^{-2}$ of $v_{\mu}+\bar{v}_{\mu}$ as a function of declination, shown for the 22-string IceCube southern and northern sky analyses (Abbasi et al. 2009a, 2009b), this 40-string analysis, and preliminary estimated sensitivities for one year for the ANTARES experiment, primarily sensitive in the $\mathrm{GeV}-\mathrm{TeV}$ energy range (Coyle 2010), and the final IceCube configuration (using the event selection based on this work for the up-going region). For the a priori source list, upper limits of Feldman-Cousins $90 \%$ confidence intervals for a flux $d \Phi / d E \propto E^{-2}$ of $v_{\mu}+\bar{v}_{\mu}$ are shown. In addition, we show the discovery potential for this work.

in Figure 18 is a preliminary comparison to the ANTARES experiment (Coyle 2010). ANTARES is primarily sensitive to $\mathrm{GeV}-\mathrm{TeV}$ energy neutrinos in the southern sky, so the coverage in energy is quite complementary to this IceCube analysis.

The Milagro TeV source stacking search resulted in a final $p$-value of $32 \%$ with best-fit $n_{s}=7.6$ (total number of signal events above background) and spectral index $\gamma=2.6$. The starburst galaxy stacking search resulted in an underfluctuation with best-fit $n_{s}=0$ and a final $p$-value of $100 \%$, since we do not allow negative values of $n_{s}$. Finally, the CGs stacking search

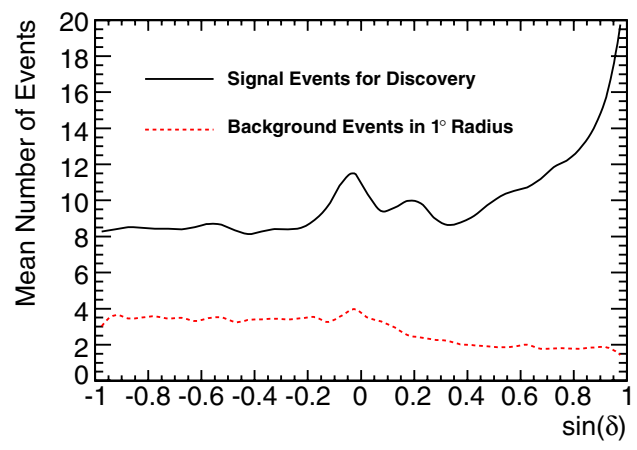

Figure 19. Mean number of $d \Phi / d E \propto E^{-2}$ signal events at the final cut level required for a discovery at $5 \sigma$ in $50 \%$ of trials and the mean number of background events in a circular bin with a radius of $1^{\circ}$ vs. sine of the declination.

yielded a final $p$-value of $78 \%$ with $n_{s}=1.8$. These results, along with sensitivities and upper limits, are summarized in Table 4.

\section{IMPLICATIONS FOR MODELS OF ASTROPHYSICAL NEUTRINO EMISSION}

The IceCube Neutrino Observatory aims to further our understanding of astrophysical phenomena, constraining models even in the absence of a detection. Figure 20 shows our sensitivity to some specific predictions. The model of Morlino et al. (2009) is for SNR RX J1713.7-3946. This analysis is largely insensitive to spectra which cut off below $100 \mathrm{TeV}$ in the southern sky. Applying this emission model at the location of the Crab 
Table 4

Results for the Stacked Source Searches

\begin{tabular}{|c|c|c|c|c|c|c|c|}
\hline Catalog & $N$ Sources & Model & $p$-value & $v_{\mu}$ Sensitivity & $v_{\mu}$ Upper Limit & $v_{\mu}+v_{\tau}$ Sensitivity & $v_{\mu}+v_{\tau}$ Upper Limit \\
\hline \multirow[t]{2}{*}{ Milagro sources } & 17 & $E^{-2}$, uniform & 0.32 & $\Phi^{90}=9.0$ & $\Phi^{90}=12.3$ & $\Phi^{90}=15.8$ & $\Phi^{90}=24.5$ \\
\hline & 6 & 6 SNR assoc. $^{\mathrm{a}}$ & $\mathrm{b}$ & & & $\mathrm{SF}=2.9$ & $\mathrm{SF}=7.2$ \\
\hline Starburst galaxies & 127 & $E^{-2}, \propto$ FIR flux & 1.00 & $\Phi^{90}=33.1$ & $\Phi^{90}=33.1$ & $\Phi^{90}=58.6$ & $\Phi^{90}=58.6$ \\
\hline \multirow[t]{4}{*}{ Clusters of galaxies } & 5 & Model $\mathrm{A}^{\mathrm{c}}$ & 0.78 & & & $\mathrm{SF}=8.4$ & $\mathrm{SF}=7.8$ \\
\hline & & Model $\mathrm{B}^{\mathrm{c}}$ & & & & $\mathrm{SF}=14.4$ & $\mathrm{SF}=12.0$ \\
\hline & & Isobaric $^{c}$ & & & & $\mathrm{SF}=13.2$ & $\mathrm{SF}=13.2$ \\
\hline & & Central $\mathrm{AGN}^{\mathrm{c}}$ & & & & $\mathrm{SF}=6.0$ & $\mathrm{SF}=6.0$ \\
\hline
\end{tabular}

Notes. Median sensitivities and upper limits at $90 \% \mathrm{CL}$ for $v_{\mu}$ and $v_{\mu}+v_{\tau}$ fluxes are given in two ways: as $\Phi^{90}$ for a spectrum $d \Phi / d E \propto E^{-2}$, i.e., the total flux from all sources $d \Phi / d E \leqslant \Phi^{90} 10^{-12} \mathrm{TeV}^{-1} \mathrm{~cm}^{-2} \mathrm{~s}^{-1}(E / \mathrm{TeV})^{-2}$, or as a scaling factor (SF) relative to the models given in the footnotes. For example, if the Central AGN model flux normalization were 6.0 times higher, we would rule it out at $90 \%$ CL. All models predict equal fluxes of tau and muon neutrinos. ${ }^{\text {a }}$ Halzen et al. (2008).

b We did not calculate an a priori $p$-value for just the six SNR associations discussed in Halzen et al. (2008), since they are included in the search over all Milagro sources. However, some of these sources were the most significant on the list. Analyzed as a single subgroup, an a posteriori $p$-value of 0.02 was found with best-fit parameters $n_{s}=15.2$ and $\gamma=2.9$. The true trial factor is incalculable since this was done after unblinding, but these remain sources of interest for future searches.

${ }^{\mathrm{c}}$ Murase et al. (2008), see Table 2.

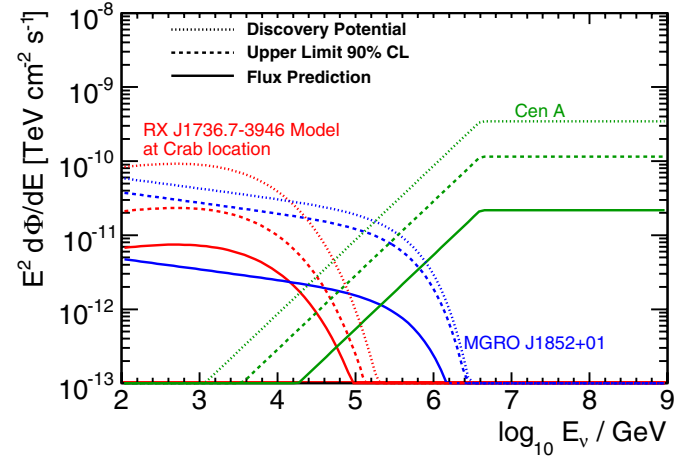

Figure 20. Differential flux of three theoretical models shown with the IceCube 40-string upper limit (90\% CL) and discovery potential in each case. Shown are the $v_{\mu}+\bar{v}_{\mu}$ predictions of Morlino et al. (2009) for SNR RX J1713.7-3946 but moved to the location of the Crab Nebula, Halzen et al. (2008) for MGRO J1852+01, and Koers \& Tinyakov (2008) for Cen A under the most optimistic condition, where the protons have a spectral index $\alpha_{p}=3$.

(A color version of this figure is available in the online journal.)

Nebula ( $\delta=22.01)$, we obtain an upper limit that rules out a flux 3.2 times higher than the prediction.

The Milagro hot spot MGRO J1852+01 is the brightest source of six SNR associations considered in Halzen et al. (2008). The stacking results were already shown in Table 4. Our upper limit for just this one brightest source is a factor of 7.9 away from excluding this model at $90 \% \mathrm{CL}$. The best fit for MGRO $\mathrm{J} 1852+01$ is to 7.0 events with $\gamma=2.9$, which increases the upper limit compared to the average background-only case.

The nearest AGN, Centaurus A (Cen A), has been discussed as a potential source of ultra-high-energy CRs in the context of results from the Pierre Auger Observatory (PAO). The pointsource likelihood fit at the location of Cen A resulted in zero signal events in this analysis, setting an upper limit that is 5.3 times higher than the $v_{\mu}$ prediction by Koers \& Tinyakov (2008) for the most optimistic case where the protons have a spectral index $\alpha_{p}=3$.

Starburst galaxies were already presented as sources of interest in Section 6.4. Recent detections (Abdo et al. 2010; Acciari et al. 2009; Aharonian et al. 2005) of very high-energy photons from the nearest luminous starburst galaxies M82 and
NGC 253, each characterized by star-forming regions with high supernova rates in the core, support the belief that the observed enhanced gamma-ray emission is due to CR interactions. Under the assumption that the $\mathrm{GeV}-\mathrm{TeV}$ photons originate from the decay of neutral pions produced when protons that are shockaccelerated by SNRs in the starburst core inelastically scatter against target hydrogen atoms with densities of the order of $10^{2} \mathrm{~cm}^{-3}$ (de Cea del Pozo et al. 2009; Persic et al. 2008), an order-of-magnitude calculation of the resulting flux of muon neutrinos based on Kelner et al. (2006) can be made. The muon neutrino upper limit from M82 is about 400 times higher than the rough prediction. For NGC 253 in the southern sky, the muon neutrino upper limit is about 6000 times higher than the prediction.

\section{CONCLUSIONS}

A search for sources of high-energy neutrinos has been performed using data taken during 2008-2009 with the 40string configuration of the IceCube Neutrino Observatory. Five searches were performed: (1) a scan of the entire sky for point sources, (2) a predefined list of 39 interesting source candidates, (3) stacking 16 sources of $\mathrm{TeV}$ gamma rays observed by Milagro and Fermi, along with an unconfirmed hot spot (17 total sources), (4) stacking 127 starburst galaxies, and (5) stacking five nearby CGs, testing four different models for the CR distribution. The most significant result of the five searches came from the all-sky scan with a $p$-value of $18 \%$. The cumulative binomial probability of obtaining at least one result of this significance or higher in five searches is $63 \%$. This result is consistent with the null hypothesis of background only.

The sensitivity of this search using 375.5 days of 40-string data already improves upon previous point-source searches in the $\mathrm{TeV}-\mathrm{PeV}$ energy range by at least a factor of two, depending on declination. The searches were performed using a data set of up-going atmospheric neutrinos (northern sky) and higher energy down-going muons (southern sky) in a unified manner. IceCube construction is now complete, with 86 strings in operation. The full IceCube detector should improve existing limits by at least another factor of two with one year of operation. Additional improvement is foreseeable 
in the down-going region by developing sophisticated veto techniques and at lower energies by using the new dense subarray, DeepCore, to its fullest potential.

We thank K. Murase for helpful discussions regarding clusters of galaxies.

We acknowledge the support from the following agencies: U.S. National Science Foundation-Office of Polar Programs, U.S. National Science Foundation-Physics Division, University of Wisconsin Alumni Research Foundation, the Grid Laboratory Of Wisconsin (GLOW) grid infrastructure at the University of Wisconsin-Madison, the Open Science Grid (OSG) grid infrastructure; U.S. Department of Energy, and National Energy Research Scientific Computing Center, the Louisiana Optical Network Initiative (LONI) grid computing resources; National Science and Engineering Research Council of Canada; Swedish Research Council, Swedish Polar Research Secretariat, Swedish National Infrastructure for Computing (SNIC), and Knut and Alice Wallenberg Foundation, Sweden; German Ministry for Education and Research (BMBF), Deutsche Forschungsgemeinschaft (DFG), Research Department of Plasmas with Complex Interactions (Bochum), Germany; Fund for Scientific Research (FNRS-FWO), FWO Odysseus Programme, Flanders Institute to encourage scientific and technological research in industry (IWT), Belgian Federal Science Policy Office (Belspo); University of Oxford, UK; Marsden Fund, New Zealand; Japan Society for Promotion of Science (JSPS); the Swiss National Science Foundation (SNSF), Switzerland; A. Groß acknowledges support by the EU Marie Curie OIF Program; J. P. Rodrigues acknowledges support by the Capes Foundation, Ministry of Education of Brazil.

\section{REFERENCES}

Abbasi, R. U., et al. 2006, ApJ, 636, 680

Abbasi, R., et al. 2009a, Phys. Rev. Lett., 103, 221102

Abbasi, R., et al. 2009b, ApJ, 701, L47

Abbasi, R., et al. 2009c, Phys. Rev., D79, 062001

Abbasi, R., et al. 2009d, Nucl. Instrum. Methods, A601, 294

Abbasi, R., et al. 2010a, Nucl. Instrum. Methods, A618, 139

Abbasi, R., et al. 2010b, Phys. Rev., D83, 012001

Abdo, A. A., et al. 2007, ApJ, 664, L91

Abdo, A. A., et al. 2009, ApJ, 700, L127

Abdo, A. A., et al. 2010, ApJ, 709, L152

Acciari, V. A., et al. 2009, Nature, 462, 770

Achterberg, A., et al. 2006a, Astropart. Phys., 26, 155

Achterberg, A., et al. 2006b, Astropart. Phys., 26, 282

Achterberg, A., et al. 2007, Phys. Rev., D75, 102001

Ackermann, M., \& Bernardini, E. 2005, in Proc. 29th ICRC, Pune, arXiv: astro-ph/0509330

Ackermann, M., et al. 2006, J. Geophys. Res., 111, D13203

Aharonian, F., et al. 2005, A\&A, 442, 177

Ahn, E.-J., Engel, R., Gaisser, T. K., Lipari, P., \& Stanev, T. 2009, Phys. Rev., D80, 094003

Ahrens, J., et al. 2004, Nucl. Instrum. Methods, A524, 169

Ambrosio, M., et al. 2001, ApJ, 546, 1038

Amsler, C., et al. 2008, Phys. Lett., B667, 1

Anchordoqui, L. A., \& Montaruli, T. 2010, Ann. Rev. Nucl. Part. Sci., 60, 129
Athar, H., Jezabek, M., \& Yasuda, O. 2000, Phys. Rev., D62, 103007

Barr, G. D., Gaisser, T. K., Lipari, P., Robbins, S., \& Stanev, T. 2004, Phys. Rev., D70, 023006

Barr, G. D., Gaisser, T. K., Robbins, S., \& Stanev, T. 2006, Phys. Rev., D74, 094009

Beacom, J. F., \& Kistler, M. D. 2007, Phys. Rev., D75, 083001

Becker, J. K. 2008, Phys. Rep., 458, 173

Becker, J. K., Biermann, P. L., Dreyer, J., \& Kneiske, T. M. 2009, arXiv:0901.1775

Bednarek, W., Burgio, G. F., \& Montaruli, T. 2005, New Astron. Rev., 49, 1

Berezinsky, V. S., Blasi, P., \& Ptuskin, V. S. 1997, ApJ, 487, 529

Berghaus, P., Birdsall, R., Desiati, P., Montaruli, T., \& Ranft, J. 2008, J. Phys. Conf. Ser., 136, 042019

Boettcher, M., Reimer, A., \& Marscher, A. 2009, ApJ, 703, 1168

Braun, J., Dumm, J., De Palma, F., Finley, C., Karle, A., \& Montaruli, T. 2008, Astropart. Phys., 29, 299

Bugaev, E., Naumov, V., Sinegovsky, S., \& Zaslavskaya, E. 1989, Nuovo Cim., C12, 41

Caprioli, D., Amato, E., \& Blasi, P. 2010, Astropart. Phys., 33, 160

Chiarusi, T., \& Spurio, M. 2010, Eur. Phys. J., C65, 649

Chirkin, D., \& Rhode, W. 2004, arXiv:hep-ph/0407075

Colafrancesco, S., \& Blasi, P. 1998, Astropart. Phys., 9, 227

Conrad, J., Botner, O., Hallgren, A., \& Perez de los Heros, C. 2003, Phys. Rev., D67, 012002

Coyle, P. 2010, in Proc. 31st ICRC, Lodz

de Cea del Pozo, E., Torres, D. F., \& Marrero, A. Y. R. 2009, ApJ, 698, 1054

Enberg, R., Reno, M. H., \& Sarcevic, I. 2008, Phys. Rev., D78, 043005

Enberg, R., Reno, M. H., \& Sarcevic, I. 2009, Phys. Rev., D79, 053006

Feldman, G. J., \& Cousins, R. D. 1998, Phys. Rev., D57, 3873

Fermi, E. 1949, Phys. Rev., 75, 1169

Gaisser, T. K., Halzen, F., \& Stanev, T. 1995, Phys. Rep., 258, 173

Gazizov, A., \& Kowalski, M. P. 2005, Comput. Phys. Commun., 172, 203

Glasstetter, R., \& Hoerandel, J. 1999, Nucl. Phys., 75, 238

Gonzalez-Garcia, M. C., Halzen, F., \& Mohapatra, S. 2009, Astropart. Phys., 31,437

Halzen, F., \& Hooper, D. 2002, Rep. Prog. Phys., 65, 1025

Halzen, F., Kappes, A., \& O'Murchadha, A. 2008, Phys. Rev., D78, 063004

Halzen, F., Kappes, A., \& O'Murchadha, A. 2009, Phys. Rev., D80, 083009

Heck, D., et al. 1998, FZKA-6019

Hill, G. C. 2003, Phys. Rev., D67, 118101

Hoerandel, J. R. 2003, Astropart. Phys., 19, 193

Honda, M., Kajita, T., Kasahara, K., Midorikawa, S., \& Sanuki, T. 2007, Phys. Rev., D75, 043006

Kang, H., Rachen, J. P., \& Biermann, P. L. 1997, MNRAS, 286, 257

Kashti, T., \& Waxman, E. 2005, Phys. Rev. Lett., 95, 181101

Kelner, S. R., Aharonian, F. A., \& Bugayov, V. V. 2006, Phys. Rev. D 74, 034018

Koers, H. B. J., \& Tinyakov, P. 2008, Phys. Rev., D78, 083009

Kotera, K., Allard, D., Murase, K., Aoi, J., Dubois, Y., Pierog, T., \& Nagataki, S. 2009, ApJ, 707, 370

Lai, H. L., Huston, J., Kuhlmann, S., Morfin, J., Olness, F., Owens, J. F., Pumplin, J., \& Tung, W. K. 2000, Eur. Phys. J., C12, 375

Learned, J. G., \& Mannheim, K. 2000, Ann. Rev. Nucl. Part. Sci., 50, 679

Lipari, P. 2006, Nucl. Instrum. Methods, A567, 405

Lundberg, J., et al. 2007, Nucl. Instrum. Methods, A581, 619

Markov, M. A., \& Zheleznykh, I. M. 1961, Nucl. Phys., 27, 385

Martin, A. D., Ryskin, M. G., \& Stasto, A. M. 2003, Acta Phys. Polon., B34, 3273

Morlino, G., Blasi, P., \& Amato, E. 2009, Astropart. Phys., 31, 376

Murase, K., Inoue, S., \& Nagataki, S. 2008, ApJ, 689, L105

Neunhoffer, T. 2006, Astropart. Phys., 25, 220

Norman, C. A., Melrose, D. B., \& Achterberg, A. 1995, ApJ, 454, 60

Persic, M., Rephaeli, Y., \& Arieli, Y. 2008, A\&A, 486, 143

Pfrommer, C., \& Ensslin, T. A. 2004, A\&A, 426, 777

Reiprich, T. H., \& Boehringer, H. 2002, ApJ, 567, 716

Thrane, E., et al. 2009, ApJ, 704, 503

Tilav, S., et al. 2010, in Proc. 30st ICRC, Lodz, arXiv:1001.0776 